Sharif University of Technology
Scientia Iranica
Transactions E: Industrial Engineering
hCIENTIA

\title{
Bi-objective scheduling for the re-entrant hybrid flow shop with learning effect and setup times
}

\author{
S.M. Mousavi ${ }^{\mathrm{a}, *}$, I. Mahdavi ${ }^{\mathrm{a}}$, J. Rezaeian ${ }^{\mathrm{a}}$, and M. Zandieh ${ }^{\mathrm{b}}$ \\ a. Department of Industrial Engineering, Mazandaran University of Science and Technology, Babol, Iran. \\ b. Department of Industrial Management, Management and Accounting Faculty, Shahid Beheshti University, G. C., Tehran, Iran. \\ Received 24 November 2015; received in revised form 15 May 2017; accepted 8 July 2017
}

\section{KEYWORDS \\ Re-entrant hybrid \\ flow shop; \\ Setup times; \\ Learning effect; \\ Multi-objective \\ problems; \\ A priori approach.}

\begin{abstract}
The production scheduling problem in hybrid flow shops is a complex combinatorial optimization problem observed in many real-world applications. The standard hybrid flow shop problem often involves unrealistic assumptions. In order to address the realistic assumptions, four additional traits were added to the proposed problem. These include the re-entrant line, setup times, position-dependent learning effects, and consideration of maximum completion time together with total tardiness as an objective function. Since the proposed problem is NP-hard, a meta-heuristic algorithm is proposed as the solution procedure. The solution procedure is categorized as an a priori approach. To show the efficiency and effectiveness of the proposed algorithm, computational experiments were carried out on various test problems. Computational results show that the proposed algorithm can obtain an effective and appropriate solution quality for our investigated problem.

(C) 2018 Sharif University of Technology. All rights reserved.
\end{abstract}

\section{Introduction}

A production unit is characterized by a multi-stage production flow shop with multiple parallel machines per production stage, usually referred to as the flexible flow shop, multi-processor flow shop, or Hybrid Flow Shop (HFS) environment. These environments have the following characteristics in common:

1. The number of stages, $g$, is at least 2 ;

2. Each stage, $t$, has $m^{t} \geq 1$ machines in parallel and in, at least, one of the stages $m^{t}>1$;

3. All $n$ jobs have the same production flow (i.e., stage 1 , stage $2, \ldots$, stage $g$ ).

Setup includes work to prepare the machine, process, or bench for product parts or the cycle. One

\footnotetext{
*. Corresponding author.
}

E-mail address: mousavi@iauns.ac.ir (S.M. Mousavi)

doi: $10.24200 /$ sci.2017.4451 of the underlying assumptions in this paper is the consideration of setup times in scheduling configurations. The setup times are classified into two types:

1. Sequence-Independent Setup Times (SIST);

2. Sequence-Dependent Setup Times (SDST).

In the former, the length of time required to do the setup depends on the job just to be processed. In the latter, the length of time required to do the setup depends on both the prior and current jobs to be processed. Allahverdi et al. [1] provided a comprehensive review of scheduling research with setup times (costs).

Another underlying assumption in this paper is the consideration of re-entrant lines in scheduling configurations. The assumption of classical HFS scheduling problems that each job visits machines in each stage only once is sometimes violated in practice. A new type of manufacturing shop, the re-entrant shop has recently attracted much attention. The Reentrant HFS (RHFS) means that there are $n$ jobs to be 
processed on $g$ stages, and every job must be processed on stages in the order of stage 1 , stage $2, \ldots$, stage $g$ for $l$ times ( $l$ is the number of repetition of jobs on the sequence of stages). Lin and Lee [2] provided a comprehensive review of the literature on scheduling problems involving re-entrant flows.

The learning effect has received considerable attention in the context of scheduling problems until recently. The learning effect in scheduling problems was first investigated by Biskup [3]. In classical scheduling, job processing and setup times are assumed constant from the first until the last job to be processed. This assumption might be unrealistic in many situations, because the productivity of a production facility (a machine, a plant, a worker, etc.) improves continuously when executed in the same or almost the same conditions. The learning effects are classified into two types:

\section{Position-based learning;}

2. The sum of processing time.

The first one assumes that the experience of the processor is equal to the number of performed jobs. In the second one, the experience provided by a job is not unary, but equal to its normal processing time. The underlying assumption in this paper is the positionbased learning effect. For further study on 'learning effect' literature in scheduling problem, refer to the complete survey, which is presented by Biskup [4].

In many real-world applications, it is often necessary to consider multiple criteria in scheduling problems. Therefore, the consideration of maximum completion time (makespan or $C_{\max }$ ) together with total tardiness as an objective function in this study is more realistic than the more common minimization of makespan or total tardiness $(\bar{T})$ separately.

The problem is configured as a multi-objective model. The first decision in a multi-objective space concerns with how to combine the search and the decision-making processes. This can be done in one of the following three ways:

1. Search and then decision-making (a posteriori approach);

2. Decision-making and then search (a priori approach);

3. Interactive search and decision-making.

In this paper, a priori approach is used to find a good quality schedule. In these methods, the solution that best satisfies the decision-maker's preferences is selected.

The HFS scheduling problem is a strongly NPhard problem. Gupta [5] showed that the two-stage HFS with more than one machine at one stage is
NP-hard. Since this problem can be considered as a specific case of the HFS, then it can be concluded that our problem is also NP-hard. The exact methods are unable to render feasible solutions even for small instances of this problem in a reasonable computational time. Therefore, this inability justifies the need for employment of a variety of heuristics and metaheuristics to solve these problems to optimality or near optimality. In this paper, a meta-heuristic algorithm is proposed to solve the scheduling problem.

The remainder of the paper is organized as follows: Section 2 gives the literature review of multiobjective HFS scheduling. Section 3 describes the problem. Section 4 introduces the proposed algorithm. Section 5 gives the computational results. Finally, Section 6 is devoted to conclusion and future research.

\section{Literature review}

The literature review section summarizes papers on the HFS problem with one or more additional features mentioned between the years 2008 to 2016 .

Jungwattanakit et al. [6] studied the flexible flow shop with several constraints to minimize a convex sum of makespan and the number of tardy jobs. They considered unrelated parallel machines and sequence/machine dependent setup times, release date, and due date as constraints in study. First, the problem was formulated by a $0-1$ Mixed Integer Programming (MIP), and then Genetic Algorithm (GA) was proposed to find the near-optimal schedule. Behnamian et al. [7] developed a multi-phase method to solve the problem of SDST HFS with the objective of minimizing the makespan as well as the sum of the earliness and tardiness of jobs. Naderi et al. [8] considered the SDST HFS problem with transportation times. They developed a Simulated Annealing (SA) to minimize both total completion time and total tardiness. Rashidi et al. [9] investigated the SDST HFS problems with unrelated parallel machines and blocking processor. They proposed the hybrid multiobjective parallel GA, which divides the population into some groups of different weights, that transforms the bi-criteria, makespan, and maximum tardiness into a single objective.

Dugardin et al. [10] considered the multi-objective RHFS scheduling problem. The scheduling objective consists of two parts: the minimization of the cycle time and the maximization of the utilization rate of the bottleneck. This problem was solved by a multiobjective GA using the Lorenz dominance relationship. Cho et al. [11] focused on the minimization of makespan and total tardiness in a RHFS. They proposed a localsearch-algorithm-based Pareto GA with the Minkowski distance-based crossover operator to achieve a good approximate Pareto solution. 
Mousavi et al. [12] considered the SDST HFS scheduling problem. In order to minimize the convex combination of the makespan and total tardiness, they proposed a meta-heuristic based on SA. In addition, Mousavi et al. [13] developed a local search to solve the above problem. Hakimzadeh Abyaneh and Zandieh [14] considered the bi-objective SDST HFS problem with limited buffers. The GA was proposed to minimize makespan and total tardiness of jobs. Pargar and Zandieh [15] investigated the SDST HFS problems with learning effect of setup times. They proposed a metaheuristic approach called water flow-like algorithm to minimize weighted sum of makespan and total tardiness.

Sheikh [16] formulated a bi-objective flexible flow shop scheduling problem with limited time lag between stages and due windows by a MIP model. A GA procedure was designed to solve this model efficiently. Tadayon and Salmasi [17] investigated group scheduling in the bi-objective flexible flow shop scheduling problem with release time and eligibility. A mathematical model and several meta-heuristic algorithms based on the Particle Swarm Optimization (PSO) algorithm were proposed to heuristically solve the research problem. Behnamian and Zandieh [18] developed a hybrid algorithm of PSO, SA, and Variable Neighborhood Search (VNS) to solve the SDST HFS scheduling with position-dependent learning effects. Fadaei and Zandieh [19] considered group scheduling in the problem of bi-objective HFS scheduling within the area of sequence-dependent family setup times. They focused on the following three multi-objective algorithms to solve the mentioned problem: multi-objective GA, subpopulation GA-II, and Non-dominated Sorting GA-II (NSGA-II).

Jolai et al. [20] investigated the bi-objective problem of no-wait two-stage flexible flow shop scheduling. The makespan together with the maximum tardiness of jobs was considered as the objective function in their study. Three bi-objective optimization methods were based on SA developed to solve scheduling problem. Luo et al. [21] studied a bi-objective HFS scheduling problem with uniform parallel machines from a new aspect of energy efficiency. In order to solve this problem, an ACO meta-heuristic was applied to optimize both makespan and electric power cost with the presence of time-of-use electricity prices. Tran and $\mathrm{Ng}[22]$ addressed the multi-objective flexible flow shop scheduling problem with limited intermediate buffers. A hybrid water flow algorithm was proposed to minimize the completion time of jobs and the total tardiness time of jobs. $\mathrm{Su}$ et al. [23] proposed a distributed co-evolutionary algorithm to minimize the makespan and total tardiness of jobs in multi-objective HFS scheduling problems.

Attar et al. [24] investigated a new multi-objective hybrid flexible flow shop problem with several useful constraints. They considered the limited waiting times between every two successive operations, unrelated parallel machines at least one stage, sequence/machine dependent setup times, and due dates of jobs as constraints in study. They proposed multi-objective PSO and strength Pareto evolutionary algorithm II to minimize the total weighted tardiness and maximum the completion times. Wang and Liu [25] considered a bi-objective HFS problem with two stages. They considered the SDST and preventive maintenance at the first stage machine as constraints in study. A multiobjective Tabu Search (TS) method was proposed to solve this integrated problem. Ying et al. [26] proposed an iterated Pareto greedy algorithm to solve a RHFS with the bi-objective of minimizing makespan and total tardiness. Mousavi and Zandieh [27] proposed a procedure based on hybrid, the simulated annealing, genetic algorithm, and local search, so-called HSA-GALS, to handle the problem of SDST HFS scheduling with the objective of minimizing the makespan and total tardiness of jobs approximately.

To the best of our knowledge, as just reviewed, bi-objective RHFS with SDST and learning effect problem have never been investigated in the scheduling problems in the literature up to now. Consequently, the scheduling models have not been developed with respect to the problem. To describe the problem in detail, a MIP model is presented. Up to now, requests, comments, and viewpoints of the decisionmakers are not included before the solution process in the scheduling problems in the literature. Consequently, a method based on an a priori approach has never been introduced as able to tackle scheduling problems within a reasonable time. To solve the problem, a meta-heuristic algorithm based on an a priori approach is proposed.

\section{Problem description}

To describe the problem in more detail, a 0-1 MIP model is presented. The indices, input parameters, decision variables, learning effect model, and the mathematical model are detailed as follows.

\subsection{Indices}

Indices, which are used to model our problem, are listed below:

$t \quad$ Index for processing stage, $t=$ $1,2,3, \cdots, g$

$i, j \quad$ Indices for jobs, $i, j=1,2,3, \cdots, n$

$k$ Index for machines at stage $t$, $k=1,2,3, \cdots, m^{t}$

$r \quad$ Index for position job on machine, $r=1,2,3, \cdots, n$ 
$l \quad$ Index for cycles performed by a job, $l=1,2,3, \cdots, L$

\subsection{Input parameters}

$n \quad$ Number of jobs to be scheduled

$g \quad$ Number of serial stages

$m^{t} \quad$ Number of identical machines at stage $t$

$L \quad$ Number of repetition of jobs on the sequence of stages

$d_{j} \quad$ Due date job $j$

$P_{j l}^{t} \quad$ Actual processing time for job $j$ at stage $t$ of layer $l$

$S_{i j l}^{t} \quad$ Actual setup time between job $j$ and job $i$ at stage $t$ of layer $l$ while job $j$ is scheduled immediately after job $i$

$S_{0 j l}^{t} \quad$ Actual setup time job $j$ at stage $t$ of layer $l$ when job $j$ is assigned to a machine at the first position

LR Learning Rate

$a_{j l}^{t} \quad$ Learning index for job $j$ at stage $t$ of layer $l$ (negative parameter)

\subsection{Decision variables}

$C_{j l}^{t} \quad$ Completion time of job $j$ at stage $t$ of layer $l$

$C_{\max } \quad$ Maximum completion time or makespan

$T_{j} \quad$ Tardiness of job $j$

$\bar{T} \quad$ Total tardiness

$X_{i j k r l}^{t} \quad 1$ if job $j$ scheduled immediately after job $i$ on machine $k$ in position $r$ at stage $t$ of layer $l$ and 0 otherwise

$X_{0 j k 1 l}^{t} \quad 1$ if job $j$ scheduled at the first position on machine $k$ at stage $t$ of layer $l$ and 0 otherwise

$X_{i 0 k n_{k, l}^{t} l}^{t} \quad 1$ if job $i$ scheduled at the last position on machine $k$ at stage $t$ of layer $l$ and 0 otherwise

$S_{0 j 1 l}^{t} \quad$ Actual setup time job $j$ at stage $t$ of layer $l$ when job $j$ is assigned to a machine at the first position

$n_{k l}^{t} \quad$ Number of jobs assigned to machine $k$ at stage $t$ of layer $l\left(\sum_{k=1}^{m^{t}} n_{k l}^{t}=n ; t=\right.$ $1,2, \cdots, g, l=1,2, \cdots, L)$

\subsection{Learning effect model}

The effect of learning on scheduling may arise in a company with similar jobs. Similar jobs may function on one machine or on parallel and identical machines for a number of customers. Generally, by processing one job after the other, the skills of the workers continuously improve, e.g., the ability to perform setups faster, to deal with the operations of the machines, or to handle raw materials, components or similar operations of the jobs at a greater pace. In this paper, scheduling problem is investigated with learning considerations, using the learning curve introduced by Biskup [3]. Now, assume that the production facility improves continuously, and that the processing time of a given job decreases as a function of its position in the sequence. As in Biskup [3], herein, it is assumed that the processing time of job $j$ at stage $t$ of layer $l$, if scheduled in position $r$, is given by Eq. (1):

$$
P_{j r l}^{t}=P_{j l}^{t} \times\left(r_{j l}^{t}\right)^{\left(a_{j l}^{t}\right)}, \quad \forall i, j, t, r, l,
$$

where $-1 \leq a_{j l}^{t} \leq 0$ is a constant learning index, given as the logarithm to base 2 of the Learning Rate (LR). In this paper, it is assumed that all machines and jobs in each stage and layer have the same learning rate $\left(a_{j l}^{t}=a\right)$. Similarly, the setup time of job $i$ to job $j$, if scheduled in position $r$ at stage $t$ of layer $l$, is given by Eq. (2):

$$
S_{i j r l}^{t}=S_{i j l}^{t} \times\left(r_{j l}^{t}\right)^{\left(a_{j l}^{t}\right)}, \quad \forall i, j, t, r, l .
$$

Therefore, decision variables related to the learning effect model are as follows:

$r_{j l}^{t} \quad$ Position job $j$ at stage $t$ of layer $l$

$P_{j r l}^{t} \quad$ The processing time for job $j$ in position $r$ at stage $t$ of layer $l$

$S_{i j r l}^{t} \quad$ The setup time of job $i$ to job $j$, scheduled in position $r$ at stage $t$ of layer $l$

\subsection{Mathematical formulation}

The problem can now be formulated as follows:

Minimize $\quad\left\{Z_{1}=C_{\max }, Z_{2}=\bar{T}\right\}$.

Subject to:

$$
\begin{aligned}
& \sum_{k=1}^{m^{t}} \sum_{r=1}^{n} \sum_{j=0, i \neq j}^{n} X_{i j k r l}^{t}=1, \quad \forall t, i, l, \\
& \sum_{k=1}^{m^{t}} \sum_{r=1}^{n} \sum_{i=0, i \neq j}^{n} X_{i j k r l}^{t}=1, \quad \forall t, j, l,
\end{aligned}
$$

$$
\sum_{j=1}^{n} X_{0 j k 1 l}^{t}=1, \quad \forall t, k, l,
$$$$
\text { or } \quad\left(\sum_{k=1}^{m^{t}} \sum_{j=1}^{n} X_{0 j k 1 l}^{t}=m^{t}, \quad \forall t, l\right) \text {, }
$$ 


$$
\begin{aligned}
& \sum_{i=1}^{n} X_{i 0 k n_{k l}^{t} l}^{t}=1, \\
& \text { or } \quad\left(\sum_{k=1}^{m^{t}} \sum_{i=1}^{n} X_{i 0 k n_{k l}^{t} l}^{t}=m^{t}, \quad \forall t, k, l,\right. \\
& X_{j j k r l}^{t}=0, \\
& \sum_{i=0, i \neq j}^{n} X_{i j k(r-1) l}^{t}-\sum_{i=1, i \neq j}^{n} X_{j i k r l}^{t} \geq 0, \\
& \forall t, k, j, r \geq 2, l,
\end{aligned}
$$$$
\sum_{i=0}^{n} \sum_{j=1, i \neq j}^{n} X_{i j k(r-1) l}^{t}-\sum_{i=0}^{n} \sum_{j=1, i \neq j}^{n} X_{i j k r l}^{t} \geq 0,
$$$$
\forall t, k, r \geq 2, l,
$$

$X_{i j k r l}^{t} \in\{0,1\}, \quad \forall t, k, r, i, j, l ; \quad i=0 ; \quad j=0$,

$S_{i j r l}^{t}=S_{i j l}^{t} \times\left(r_{j l}^{t}\right)^{\left(a_{j l}^{t}\right)}, \quad \forall i, j, t, r, l$,

$P_{j r l}^{t}=P_{j l}^{t} \times\left(r_{j l}^{t}\right)^{\left(a_{j l}^{t}\right)}, \quad \forall i, j, t, r, l$,

$C_{j l}^{t}-C_{i l}^{t} \geq S_{i j r l}^{t}+P_{j r l}^{t}+\left(\sum_{k=1}^{m^{t}} X_{i j k r l}^{t}-1\right) M$

$$
\forall t, i, j, r \geq 2, l ; \quad i \neq j
$$

$C_{j l}^{t} \geq 0$

$$
\forall t, j, l,
$$

$$
\begin{gathered}
C_{j l}^{t}-C_{j l}^{t-1} \geq \sum_{k=1}^{m^{t}} \sum_{i=1}^{n} S_{i j r l}^{t} X_{i j k r l}^{t}+\sum_{k=1}^{m^{t}} S_{0 j r l}^{t} X_{0 j k r l}^{t} \\
+P_{j r l}^{t},
\end{gathered}
$$

$$
C_{\max } \geq C_{j L}^{g}, \quad \forall j,
$$

$T_{j} \geq C_{j L}^{g}-d_{j}, \quad \forall j$,

$$
T_{j} \geq 0, \quad \forall j,
$$

$$
\begin{array}{ll}
\bar{T}=\sum_{j=1}^{n} T_{j}, & \\
X_{i j k 1 l}^{t}=0, & \forall i \geq 1, j, k, t, l ; \quad i \neq j, \\
X_{0 j k r l}^{t}=0, & \forall r \geq 2, j, k, t, l, \\
X_{i 0 k r l}^{t}=0, & \forall r<n_{k l}^{t}, i, k, t, l, \\
\sum_{i=0}^{n} \sum_{j=1}^{n} X_{i j k r l}^{t}=n_{k l}^{t}, & \forall k, l, t, \\
\sum_{k=1}^{m^{t}} n_{k l}^{t}=n, & \forall t, l .
\end{array}
$$

Eq. (3) describes the objective function. Constraint sets (4) and (5) ensure that only one job is assigned to each sequence position at each stage and layer. Constraint sets (6) and (7) ensure that only one job will be assigned to the first and last positions, respectively, on each machine at each stage and layer. Constraint sets (6) and (7) (in parenthesis) show that $m^{t}$ machines are scheduled at each stage and layer. Constraint set (8) assures that, after the job has been finished at any stage, it cannot be reprocessed at the same stage. Constraint set (9) is a flow balance constraint, guaranteeing that jobs are performed in a well-defined sequence, and ensuring that each job has a predecessor and a successor on the machine where the job is processed. That is, if job $j$ is processed directly after job $i$ on machine $k$ in position $r-1$ at stage $t$ of layer $l$, job $i^{\prime}$, which is the successor of the job $j$, should be processed in position $r$ on machine $k$ at stage $t$ of layer $l$. Constraint set (10) ensures that the position on each machine should be filled in sequence. Constraint set (9) is complementary to Constraint (10). Constraint set (11) specifies decision variables $X_{i j k r l}^{t}$ as binary variables. Constraint set (12) modifies setup time between job $j$ and job $i$ in position $r$ at stage $t$ of layer $l$ with respect to learning effect. Constraint set (13) modifies processing time for job $j$ in position $r$ at stage $t$ of layer $l$ with respect to learning effect. Constraint set (14) is a set of disjunctive constraints. It states that if jobs $i$ and $j$ are scheduled on the same machine at a particular stage with job $i$ scheduled before job $j$, then job $i$ must complete the processing before job $j$ can begin. This constraint set forces job $j$ to follow job $i$ by at least the processing time of job $j$ plus the setup time from $i$ to $j$ if job $j$ is immediately scheduled after job $i$. The value of $M$ is set to a very large constant. Constraint set (15) ensures that the completion time of every job at each stage and layer is a non-negative value. Constraint set (16) specifies the conjunctive precedence 
constraints for the jobs, stating that a job cannot start its processing at stage $t$ before it is finished at stage $t-1$. Constraint set (17) links the makespan decision variable $\left(C_{\max }=\max \left\{C_{j}^{g}, j=1, \cdots, n\right\}\right)$. Constraint sets (18) and (19) determine the correct value of the tardiness $\left(T_{j}\right)$. Constraint set (18) determines the correct value of the lateness, and constraint set (19) specifies only the positive lateness as the tardiness $\left(T_{j}=\max \left\{C_{j}^{g}-d_{j}, 0\right\}\right)$. Constraint set (20) links the total tardiness decision variable. Constraint sets (17) and (20) represent two criteria of the objective function complementary with other constraints. Constraint sets (21) to (23) provide limits on the decision variables (the undefined variables' values become equal to 0 ). Constraint sets (24) and (25) calculate the number of jobs assigned to each machine.

\section{The proposed algorithm}

In this paper, VNS based on an a priori approach, namely VNS-PA, is proposed for solving this biobjective optimization problem. The proposed algorithm is categorized as a local search-based algorithm armed with systematic neighborhood search structures.

In a nutshell, VNS algorithm starts from an initial solution and manipulates it through a two-nested loop. The outer loop works as a refresher reiterating the inner loop, while the inner loop carries out the major search. The inner loop iterates as long as it keeps improving the solutions. Once an inner loop is completed, the outer loop reiterates until the termination condition is met.

The solution procedure is categorized as an a priori approach. In the case of a priori methods, the decision-maker must specify her or his preferences, hopes, and opinions before the solution process. Therefore, a decision-maker along with his or her preference structure is required. For this reason, several provisions are designed to express the views of decision-makers. In the following subsection, the provisions in detail are described.

\subsection{The designed provisions}

In many studies, the aim is to find a good quality schedule for their proposed problem that minimizes a convex combination of objective functions (i.e., makespan and total tardiness). Therefore, for a solution, $x$, the total objective function is represented as follows:

Total objective function $=$ minimizing $f(x)$,

$$
\begin{aligned}
& f(x)=\lambda_{1} \times f_{1}(x)+\lambda_{2} \times f_{2}(x), \\
& f_{1}(x)=\text { makespan; } f_{2}(x)=\text { total tardiness; } \\
& \lambda_{1}+\lambda_{2}=1,
\end{aligned}
$$

where $\lambda_{1}, \lambda_{2} \geq 0$ values are the weighting coefficients representing the relative importance of makespan and the total tardiness. The idea behind $\lambda$ values is to balance both objectives.

According to a priori approach, the preferences for each objective are set by the decision-makers; then, one solution satisfying these preferences has to be found. It must be said that all requests, comments, and viewpoints of the decision-makers are not included in the total objective function (Eq. (26)). Instead of this function, a new objective function must be designed in terms of requests, comments, and viewpoints of the decision-makers. For this reason, several provisions are designed to express the views of decision-makers. Then, a new objective function is designed according to these provisions. The provisions designed in this paper are now described as follows:

1. The first provision: The decision-makers require schedules with respect to the trade-off between various objectives. Figure 1 presents the acceptable trade-off between the objectives by angle $(\alpha)$. The angle of the $i$ th neighborhood solution $\left(x_{i}\right)$, called $\alpha_{x_{i}}$, is computed as given in Eq. (27), and the first provision is designed as given in Eq. (28). According to the first provision, $\alpha_{x_{i}}$ at the interval of 35 to $55(45 \pm 10)$ degrees is proper and condition is satisfied:

$$
\alpha_{x_{i}}= \begin{cases}\arctan \left(\frac{\left(\frac{f_{2}\left(x_{i}\right)}{f_{2}}\right)}{\left(\frac{f_{1}\left(x_{i}\right)}{f_{1}}\right)}\right) & \forall i \text { if } f_{2}>0 \\ \arctan \left(\frac{\left(\frac{1+f_{2}\left(x_{i}\right)}{1+f_{2}}\right)}{\left(\frac{f_{1}\left(x_{i}\right)}{f_{1}}\right)}\right) & \forall i \text { if } f_{2}=0\end{cases}
$$

If $35 \leq \alpha_{x_{i}} \leq 55, \quad \forall i$

Then $\quad X_{1}^{i}=1, \quad$ Else $\quad X_{1}^{i}=0$,

where $f_{1}\left(x_{i}\right)$ and $f_{2}\left(x_{i}\right)$ are the makespan and total tardiness of the $i$ th neighborhood solution,

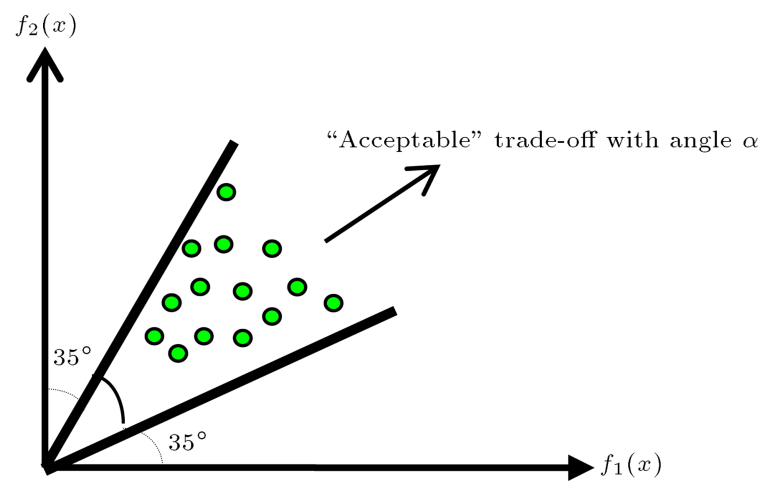

Figure 1. A range of angle as an acceptable trade-off between the two objectives. 
respectively. It is noted that several neighborhood solutions derived from the current solution $\left(x_{0}\right)$ are generated. $f_{1}$ and $f_{2}$ are the lowest observed makespan and total tardiness values, respectively, which can be updated after each iteration. To prevail over the trap of dealing with different measurement sizes of objective values, the value of each objective function should be normalized by dividing the actual objectives' values into the minimum objectives. In this respect, one is added to the denominator and numerator when $f_{2}$ is equal to $0\left(f_{2}=0\right)$;

2. The second provision: The hope of decision-makers is to find schedules close to the ideal point $(0$, $0)$. For this reason, the obtained solutions should converge towards the ideal point. Figure 2 presents the convergence of the ideal point by distance $(e d)$. The distance between the ideal point and the $i$ th neighborhood solution, called $e d_{x_{i}}$, is computed as given in Eq. (29), and the second provision is designed as given in Eq. (30). According to the second provision, if $e d_{x_{i}}$ has lower value of distance between the ideal point and current solution $\left(e d_{x_{0}}\right)$, then the condition for the corresponding solution is satisfied. Parameter definitions are presented as before.

$$
e d_{x_{i}}=\left\{\begin{array}{l}
\sqrt{\left(\frac{f_{1}\left(x_{i}\right)}{f_{1}}\right)^{2}+\left(\frac{f_{2}\left(x_{i}\right)}{f_{2}}\right)^{2}} \quad \forall i, \\
\text { if } f_{2}>0 \\
\sqrt{\left(\frac{f_{1}\left(x_{i}\right)}{f_{1}}\right)^{2}+\left(\frac{1+f_{2}\left(x_{i}\right)}{1+f_{2}}\right)^{2}} \quad \forall i, \\
\text { if } f_{2}=0
\end{array}\right.
$$

$$
\begin{aligned}
& \text { If } \quad e d_{x_{i}} \leq e d_{x_{0}}, \quad \forall i, \\
& \text { Then } \quad X_{2}^{i}=1, \quad \text { Else } \quad X_{2}^{i}=0 .
\end{aligned}
$$

3. The third provision: Suppose that there is a basic solution. The decision-makers are interested in accepting new solutions, compared to basic solution, only if they provide a better value, or at least one objective, i.e., $f_{1}(x)$ or $f_{2}(x)$. For a

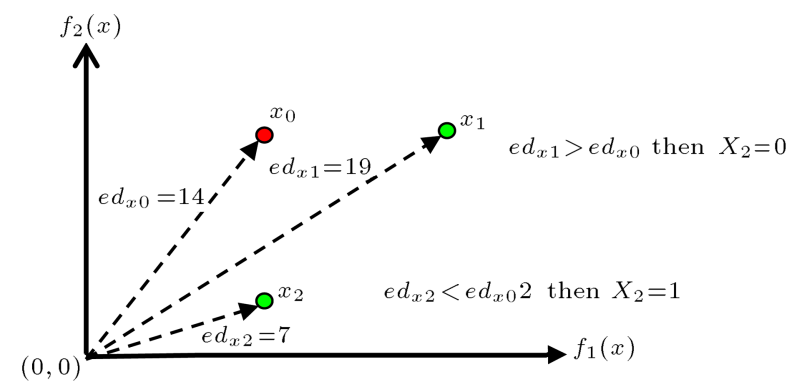

Figure 2. A hypothetical example of distance. bi-objective problem, this criterion is defined as given in Eq. (31). This criterion is one of the simplest acceptance criteria defined with decisionmakers' comments. Similar to the mentioned criterion, the third provision is designed as given in Eq. (32). According to the third provision, if at least one objective of $i$ th neighborhood solution $\left(f_{1}\left(x_{i}\right)\right.$ or $\left.f_{2}\left(x_{i}\right)\right)$ has lower value of the current solution $\left(f_{1}\left(x_{0}\right)\right.$ or $\left.f_{2}\left(x_{0}\right)\right)$, then the condition for the corresponding solution is satisfied:

$$
\begin{aligned}
& f_{1}\left(x_{i}\right)<f_{1}\left(x_{0}\right), \quad \text { or } \quad f_{2}\left(x_{i}\right)<f_{2}\left(x_{0}\right), \quad \forall i, \\
& \text { If } f_{1}\left(x_{i}\right)<f_{1}\left(x_{0}\right), \quad \text { or } f_{2}\left(x_{i}\right)<f_{2}\left(x_{0}\right), \quad \forall i \text {, } \\
& \text { Then } X_{3}^{i}=1, \quad \text { Else } X_{3}^{i}=0 \text {. }
\end{aligned}
$$

4. The fourth provision: Researchers use Relative Percentage Deviation (RPD) as a common performance measure. This criterion is shown in Eq. (33):

$$
\mathrm{RPD}=\frac{\mathrm{Alg}_{\mathrm{sol}}-\min _{\mathrm{sol}}}{\min _{\mathrm{sol}}}
$$

where $A \lg _{\text {sol }}$ denotes the objective function value obtained for a given algorithm, $\min _{\text {sol }}$ indicates the best obtained value for objective function. It is clear that lower values of RPD are preferable. For a bi-objective problem, the RPD of the $i$ th neighborhood solution, called $\mathrm{RPD}_{x_{i}}$, is computed as given in Eq. (34). Based on the mentioned criterion, the fourth provision is designed as given in Eq. (35). According to the fourth provision, if $\mathrm{RPD}_{x_{i}}$ has lower value of the current solution $\left(\mathrm{RPD}_{x_{0}}\right)$, then the condition for the corresponding solution is satisfied. Parameter definitions are presented as before:

$$
\begin{aligned}
& \operatorname{RPD}_{x_{i}}=\left\{\begin{array}{c}
\frac{f_{1}\left(x_{i}\right)-f_{1}}{f_{1}}+\frac{f_{2}\left(x_{i}\right)-f_{2}}{f_{2}} \quad \forall i, \\
\text { If } f_{2}>0 \\
\frac{f_{1}\left(x_{i}\right)-f_{1}}{f_{1}}+\frac{f_{2}\left(x_{i}\right)-f_{2}}{1+f_{2}} \quad \forall i, \\
\text { If } f_{2}=0
\end{array}\right. \\
& \text { If } \operatorname{RPD}_{x_{i}}<\operatorname{RPD}_{x_{0}}, \quad \forall i, \\
& \text { Then } X_{4}^{i}=1, \quad \text { Else } \quad X_{4}^{i}=0
\end{aligned}
$$

5. The fifth provision: One of the other views of decision-makers is to minimize a convex combination of objective functions. For a bi-objective problem, a convex combination of the $i$ th neighborhood solution, called $f\left(x_{i}\right)$, is computed in Eq. (36). Based on the mentioned function, the fifth provision is designed as given in Eq. (37). According to 
the fifth provision, if $f\left(x_{i}\right)$ has lower value of the current solution $\left(f\left(x_{0}\right)\right)$, then condition for the corresponding solution is satisfied:

$$
\begin{aligned}
& f\left(x_{i}\right)=\left(\lambda \times f_{1}\left(x_{i}\right)+(1-\lambda) \times f_{2}\left(x_{i}\right)\right), \\
& \quad \forall i, \\
& \text { If } f\left(x_{i}\right)<f\left(x_{0}\right), \quad \forall i, \\
& \text { Then } \quad X_{5}^{i}=1, \quad \text { Else } \quad X_{5}^{i}=0 .
\end{aligned}
$$

Note that the objectives are not normalized. Consequently, this criterion is sensitive to increasing and decreasing in the objective function with a larger value. The application of this criterion is important when objective values of the new solutions are close to the current solution. In the following, an example is provided to clarify. Suppose that the current solution is $(10,1500)$, and new solutions, $x_{1}$ and $x_{2}$, are $(9,1505)$ and (11, 1495), respectively. According to the third provision, both new solutions are acceptable. If $\lambda$ is set equal to 0.5 , then objective functions $(f(x))$ are calculated as follows: $f\left(x_{0}\right)=755, f\left(x_{1}\right)=757$, and $f\left(x_{2}\right)=753$. According to the fifth provision, only $x_{2}$ is acceptable;

6. The sixth provision: Similar to the fifth provision, the sixth provision is designed. Instead of actual values, the normalized objectives have been used in the provision. The normalized objectives are computed as given in Eq. (38):

$$
\begin{aligned}
& f_{1}^{\prime}(x)=\frac{f_{1}}{f_{1}(x)}, \\
& f_{2}^{\prime}(x)=\left\{\begin{array}{ll}
\frac{f_{2}}{f_{2}(x)} & \text { if } f_{2}>0 \\
\frac{1+f_{2}}{1+f_{2}(x)} & \text { if } f_{2}=0 \text { or } f_{2}(x)=0
\end{array}\right\} .
\end{aligned}
$$

The one is added to the denominator and numerator when the total tardiness or minimum total tardiness is equal to 0 .

The total objective function of the $i$ th neighborhood solution, called $f^{\prime}\left(x_{i}\right)$, is computed as given in Eq. (39). Based on the mentioned function, the sixth provision is designed as given in Eq. (40). According to the sixth provision, if $f^{\prime}\left(x_{i}\right)$ has lower value of the current solution $\left(f^{\prime}\left(x_{0}\right)\right)$, then the condition for the corresponding solution is satisfied:

$$
\begin{aligned}
& f^{\prime}\left(x_{i}\right)=\left(\lambda \times f_{1}^{\prime}\left(x_{i}\right)+(1-\lambda) \times f_{2}^{\prime}\left(x_{i}\right)\right)^{-1}, \\
& \text { If } f^{\prime}\left(x_{i}\right)<f^{\prime}\left(x_{0}\right) \quad \forall i,
\end{aligned}
$$$$
\text { Then } \quad X_{6}^{i}=1 \quad \text { Else } X_{6}^{i}=0 \text {. }
$$

7. The seventh provision: The total deviation of each new solution of the current solution, called Deviation $_{x_{i}}$ is computed as given in Eq. (41). The result of this deviation can be positive or negative. Two cases represent a negative value as follows:

(a) Both objectives are worse;

(b) One of the objectives is better and the other is worse;

however, the negative deviation will dominate the positive deviation. Two cases represent a positive value as follows:

(a) Both objectives are better;

(b) One of objectives is better and the other is worse;

however, the positive deviation will dominate the negative deviation. Consequently, the positive results are more suitable. In fact, decision-makers are interested in achieving new solutions with the positive values of the total deviation. Based on the mentioned criterion, the seventh provision is designed as given in Eq. (42). According to the seventh provision, if Deviation $_{x_{i}}$ has a positive value, then the condition for the corresponding solution is satisfied:

$$
\begin{aligned}
& \text { Deviation }_{x_{i}}=\frac{f_{1}\left(x_{0}\right)-f_{1}\left(x_{i}\right)}{f_{1}\left(x_{0}\right)}+\frac{f_{2}\left(x_{0}\right)-f_{2}\left(x_{i}\right)}{\left(1+f_{2}\left(x_{0}\right)\right)}, \\
& \forall i, \\
& \text { If deviation } x_{i}>0, \quad \forall i, \\
& \text { Then } X_{7}^{i}=1, \quad \text { Else } \quad X_{7}^{i}=0 .
\end{aligned}
$$

Now, the difference between the third and seventh provisions is described. In the third provision, decision-makers accept new solutions only if they provide a better value, even at least one objective. In the seventh provision, first, at least one of objectives provide a better value. Then, the positive deviation dominates the negative deviation. In the progress, the difference between the fourth and seventh provisions is described. In the fourth provision, old and new solutions are measured to a minimum value separately. In the seventh provision, old and new solutions are measured directly together;

8. The proposed objective function: Based on the seven provisions described, a new objective function is defined in terms of requests, comments, and viewpoints of the decision-makers as follows (Eq. (43)): 
Total objective function $=$ maximizing $Z$,

$$
\begin{aligned}
& Z^{i}=X_{1}^{i}+X_{2}^{i}+X_{3}^{i}+X_{4}^{i}+X_{5}^{i}+X_{6}^{i}+X_{7}^{i}, \\
& \quad \forall i
\end{aligned}
$$$$
\text { s.t.: }
$$$$
X_{j}^{i}= \begin{cases}1 & \text { If } j \text { th condition of neighborhood } \\ & \text { solution } i \text { th is satisfied } \\ 0 & \text { Otherwise }\end{cases}
$$$$
j=1,2, \cdots, 7 \text {. }
$$

Some interesting points are presented as follows:

- First, the objective function is converted from the minimizing into the maximizing;

- Second, seven additional traits are added to the objective function;

- Third, decision-makers express the features added to the objective function;

- Fourth, the objective function will serve as a "template" to/from which the assumptions and constraints will be added or removed to describe different objective function variants.

\subsection{The proposed algorithm pseudo code}

The pseudo code of the VNS-PA algorithm applied in this paper is now presented as follows.

\section{Initialization}

- Encoding: Integer coding is used in this research for the representation of a solution. In this kind of representation, a single row array of the size equal to the number of the jobs to be scheduled is formed. The value of the first element of the array shows which job is scheduled first. The second value shows the job scheduled, and so on. For an example, a solution is generated according integer coding as [ [ $\left.\begin{array}{lllll}3 & 1 & 4 & 2 & 5\end{array}\right]$ for a problem with five jobs $(n=5)$;

- Input parameters: Maximum number of iteration of inner loop ( $\left.\max _{-} i t\right)$; the weighting coefficients $(\lambda \in\{0.25,0.5,0.75\})$;

- Draw an initial solution, $x_{0}$ : The initial solution is generated in a random way from the search space;

- Evaluate $\boldsymbol{f}_{\mathbf{1}}\left(\boldsymbol{x}_{0}\right)$ and $\boldsymbol{f}_{\mathbf{2}}\left(\boldsymbol{x}_{0}\right): f_{1}\left(x_{0}\right)$ is the makespan; $f_{2}\left(x_{0}\right)$ is the total tardiness of initial solution;

- Set: $q=1$, archive $(q)=\left\{x_{0}\right\}, f_{1}=f_{1}\left(x_{0}\right)$, and $f_{2}=f_{2}\left(x_{0}\right)$.
For $i t=1$ : ax $_{\text {it }}$, \% maximum number of iteration inner loop is $\max \_i t$

$$
N S S=1
$$

While $N S S<4$

Follow Steps 1 to 3 , respectively.

Step 1: Generate neighborhood solutions:

If $N S S=1$, perform the swap move on $x_{0}$ and generate $n$ solutions;

If $N S S=2$, perform the shift move on $x_{0}$ and generate $n$ solutions;

If $N S S=3$, perform the inversion move on $x_{0}$ and generate $n$ solutions;

Evaluate $f_{1}\left(x_{i}\right)$ and $f_{2}\left(x_{i}\right)$ as new solutions in the neighborhood of $x_{0}$;

Update $f_{1}$ and $f_{2}$ as $f_{1}=\min \left\{f_{1}, f_{1}\left(x_{i}\right) i=\right.$ $1,2, \cdots, n\}, f_{2}=\min \left\{f_{2}, f_{2}\left(x_{i}\right) i=1,2, \cdots, n\right\}$.

Step 2: Consider provisions and calculate $Z$ for each neighborhood solution.

Consider conditions (Eqs. (28), (30), (32), (35), (37), (40), and (42)) for each neighborhood solution:

If $35 \leq \alpha_{x_{i}} \leq 55, \quad \forall i$

Then $\quad X_{1}^{i}=1, \quad$ Else $X_{1}^{i}=0$,

If $e d_{x_{i}} \leq e d_{x_{0}}, \quad \forall i$,

Then $X_{2}^{i}=1, \quad$ Else $X_{2}^{i}=0$,

If $\left\{\begin{array}{l}f_{1}\left(x_{i}\right)<f_{1}\left(x_{0}\right) \\ \text { or } \\ f_{2}\left(x_{i}\right)<f_{2}\left(x_{0}\right)\end{array} \quad \forall i\right.$,

Then $\quad X_{3}^{i}=1, \quad$ Else $\quad X_{3}^{i}=0$

If $\operatorname{RPD}_{x_{i}}<\operatorname{RPD}_{x_{0}}, \quad \forall i$,

Then $\quad X_{4}^{i}=1, \quad$ Else $\quad X_{4}^{i}=0$,

If $f\left(x_{i}\right)<f\left(x_{0}\right), \quad \forall i$,

Then $X_{5}^{i}=1, \quad$ Else $X_{5}^{i}=0$,

If $f^{\prime}\left(x_{i}\right)<f^{\prime}\left(x_{0}\right), \quad \forall i$,

Then $\quad X_{6}^{i}=1, \quad$ Else $\quad X_{6}^{i}=0$,

If Deviation $x_{i}>0, \quad \forall i$,

Then $\quad X_{7}^{i}=1, \quad$ Else $\quad X_{7}^{i}=0$. 
Calculate objective function $Z$ (Eq. (43)) for each neighborhood solution:

$$
\begin{aligned}
& Z^{i}=X_{1}^{i}+X_{2}^{i}+X_{3}^{i}+X_{4}^{i}+X_{5}^{i}+X_{6}^{i}+X_{7}^{i}, \\
& \quad \forall i \\
& \text { s.t.: }
\end{aligned}
$$

$$
\underset{j=1,2, \cdots, 7}{X_{j}^{i}}= \begin{cases}1 & \begin{array}{l}
\text { If } j \text { th condition of } \\
\text { neighborhood solution } i \text { th } \\
\text { is satisfied }
\end{array} \\
0 & \text { Otherwise }\end{cases}
$$

Step 3: Decision-making

If $\exists k$ that $Z^{k}=\max \left(Z^{i} ; \forall i\right) \geq 4$ (accept

$57.1 \%$ of the cited conditions), Then

$x_{0}=$ the corresponding solution with maximal number of provisions (or $x_{0} \leftarrow x_{k}$ ) $q=q+1$

Archive $(q)=\left\{x_{k}\right\}$

$N S S=N S S$

Else

End If

$$
N S S=N S S+1
$$

End For

Select one of the archived solutions according to provisions.

\section{Computational experiments}

This section contains the method for generating data sets, runs these data sets by the proposed algorithm, full enumeration algorithm, and algorithm in the literature, and expresses the results of the validation of the proposed model, the proposed algorithm; then, the results of the efficiency of the proposed algorithm are presented.

\subsection{Test problems}

The data required for a problem consist of the number of re-entrants, jobs, machines per stage, stages, processing times, due dates, setup times, and learning indices. The designing range of the levels of each factor is illustrated, as shown in Table 1. The number of machines, processing times, and setup times are randomly generated from a discrete uniform distribution, as described in Table 1 . This table includes 4 categories of problems: (1) special small, (2) small, (3) medium, and (4) large problems. Special small problems are designed to assess the validity of the proposed algorithm. The specific name is given to these problems because they cover small problems of single machine, parallel machine, flow shop, and two-stage HFS. To demonstrate the effectiveness of the proposed VNS-PA compared to algorithm in the literature, the experiments are conducted on three sizes of problems: small, medium, and large. The twenty-four problems are produced for the special small problems. Ten problems are produced for the small, medium, and large problems. Learning indices -0.152 and -0.514 are selected with respect to the learning curve of $90 \%$ and $70 \%$, respectively. In general, all problems are tested with regard to the level of learning indices. To generate due dates of all $n$ jobs, the following steps are proposed:

- Compute the total processing time of each job on all $g$ stages:

$$
P_{j}=\sum_{l=1}^{L} \sum_{t=1}^{g} P_{j l}^{t}, \quad \forall j \in n .
$$

\begin{tabular}{|c|c|c|c|c|}
\hline \multirow{2}{*}{ Factor } & \multicolumn{4}{|c|}{ Levels } \\
\hline & Special small & Small & Medium & Large \\
\hline Number of jobs $(n)$ & $5 ; 7 ;$ and10 & $10 ; 15 ;$ and 20 & $25 ; 30 ;$ and 35 & $40 ; 50 ;$ and 60 \\
\hline Number of stages $(g)$ & $1 ;$ and 2 & $5 ; 7 ;$ and 10 & $10 ; 12 ;$ and 15 & $15 ; 17 ;$ and 20 \\
\hline Number of re-entrants $(L)$ & $1 ;$ and 2 & $1 ;$ and 2 & $2 ;$ and 3 & $3 ;$ and 4 \\
\hline Number of machines $\left(m^{t}\right)$ & $1 ;$ and 3 & Uniform $(1,3)$ & Uniform $(1,6)$ & Uniform $(1,9)$ \\
\hline Processing times $(P)$ & Uniform $(10,20)$ & Uniform $(10,20)$ & Uniform $(10,40)$ & Uniform $(10,100)$ \\
\hline Setup times $(S)$ & Uniform $(3,6)$ & Uniform $(3,6)$ & Uniform $(5,10)$ & Uniform $(11,22)$ \\
\hline
\end{tabular}

- Compute average setup time for all possible subsequent jobs and sum it for all $g$ stages:

$$
S_{j}=\sum_{l=1}^{L} \sum_{t=1}^{g}\left(\frac{\sum_{k=1}^{n} S_{k j l}^{t}}{n}\right), \quad \forall j \in n .
$$

Table 1. Factors and their levels. 
- Determine a due date for each job:

$$
\begin{aligned}
& d_{j}=\left(P_{j}+S_{j}\right) \times\left(\frac{\max \left(\begin{array}{c}
m^{t} \\
t \in g
\end{array}\right)}{g}\right) \times(1+\text { random } \times 3), \\
& \forall j \in n,
\end{aligned}
$$

where random is a random number from a uniform distribution over range $(0,1)$.

\subsection{The validation of proposed model}

To demonstrate the validation of the proposed model, the experiments were conducted on five special small problems. Each example is solved by the full enumeration algorithm and LINGO software. Solving a problem with enumeration algorithm includes trying all the possibilities that exist with manual calculations. Details of the special small-sized problems and results are shown in Table 2. Because the process of the solution is time consuming, the runtime was limited to two hours.

It is obvious from the table that the optimal solution has been obtained for the first problem. This result demonstrates the ability of the proposed approach to model the problem and find an optimal solution. Due to time constraints, a gap between the optimal solution and obtained solution exists for other problems. The results show that error increases significantly when the dimensions are slightly larger. Thus, meta-heuristic methods must be used to solve problems.

\subsection{The validation of the proposed algorithm}

To demonstrate the validation of the proposed VNSPA, the experiments were conducted on special small problems. The full enumeration algorithm is used to find the optimal solution to every problem. Details of special small-sized problems and results are shown in Table 3. According to the table, the first column indicates the abbreviation codes of each test problem, the second and third columns describe the details of problems (number of jobs $\times$ number of stages $\times$ number of re-entrants, and number of machines per stage), the fourth describes learning indices, the fifth describes the best value of objectives for the proposed objective function, and the last column describes the average CPU time (second unit).

It is noticeable that the maximum number of iteration of inner loop, called max_it, is the only parameter of the proposed algorithm and set $\max \_i t=$ 10. Based on the results given in Table 3 , the following observations can be drawn.

Due to the proposed objective function, the proposed algorithm is able to find the optimal schedule in $93.75 \%$ of the cases. This result indicates that the proposed algorithm has very high reliability (excellent performance) to solve the problems. The proposed algorithm is able to solve the problems in the length of the interval from 0.2340 to 2.9484 seconds. The full enumeration algorithm has spent the interval from 0.0780 to 2713.5281 seconds. This result indicates that the proposed algorithm has a significant speed in solving the problem. The proposed algorithm is able to find the optimal schedule in $58.33 \%$ of the cases faster than the full enumeration algorithm.

\subsection{Numerical result}

To demonstrate the efficiency of the proposed VNSPA, the Simulated Annealing (SA) algorithm proposed by Mousavi et al. [12] is used. It is noticeable that all of algorithms are implemented in MATLAB 2009a, which is a special mathematical computation language and run on a PC with $2.30 \mathrm{GHz}$ Intel Core and 4 GB of RAM memory. To show the efficiency and effectiveness of the proposed algorithm in comparison with a SA, computational experiments were carried out on various test problems (i.e., small, medium, and large). The three replications of each problem size have been performed since there are some random conditions when applying the algorithm.

Tables 4 to 6 show the results of the implementation of algorithms on various problems. In addition, the first column indicates the abbreviation codes of each test problem, the second and third columns describe the weights of sets $\{0.25,0.5$, and 0.75$\}$ and learning indices of sets $\{-0.152$ and -0.514$\}$, the fourth column describes the best combination of objectives for each algorithm, the fifth column describes the value of the proposed objective function (Eq. (42)) for each algorithm, compared to their solutions in the fourth

\begin{tabular}{|c|c|c|c|c|c|c|}
\hline \multirow{2}{*}{$\begin{array}{c}\text { Test } \\
\text { problem }\end{array}$} & \multicolumn{2}{|c|}{ Details of problems } & \multirow{2}{*}{$a^{t}$} & \multirow{2}{*}{$\begin{array}{l}\text { Optimal solution } \\
\quad\left(f_{1}(x), f_{2}(x)\right)\end{array}$} & \multirow{2}{*}{$\begin{array}{l}\text { LINGO results } \\
\left(f_{1}(x), f_{2}(x)\right)\end{array}$} & \multirow{2}{*}{ Gap \% } \\
\hline & $n \times g \times L$ & $m^{t}$ & & & & \\
\hline 1 & $10 \times 1 \times 1$ & 1 & -0.152 & $(151.2996,351.0591)$ & Optimal solution & 0 \\
\hline 2 & $10 \times 2 \times 1$ & 1,1 & -0.152 & $(171.3400,474.0023)$ & $(185.1399,573.1197)$ & 17.50 \\
\hline 3 & $10 \times 2 \times 1$ & 3,3 & -0.152 & $(78.3015,0)$ & $(88.7881,38.9454)$ & 63.13 \\
\hline 4 & $10 \times 2 \times 2$ & 3,3 & -0.152 & $(139.3810,46.0765)$ & $(159.2371,192.8482)$ & 89.85 \\
\hline 5 & $10 \times 2 \times 3$ & 3,3 & -0.152 & $(242.6945,516.8420)$ & No solution & Infinite \\
\hline
\end{tabular}

Table 2. Details of special small-sized problems and results of LINGO. 
Table 3. Details of special small-sized problems and results of algorithms.

\begin{tabular}{|c|c|c|c|c|c|c|c|}
\hline \multirow{2}{*}{$\begin{array}{c}\text { Test } \\
\text { problem }\end{array}$} & \multirow{2}{*}{$n \times g \times L$} & \multirow{2}{*}{$m^{t}$} & \multirow{2}{*}{$a^{t}$} & \multicolumn{2}{|c|}{$\begin{array}{c}\text { Best combination } \\
\left(f_{1}(x), f_{2}(x)\right)\end{array}$} & \multicolumn{2}{|c|}{$\begin{array}{l}\text { CPU time } \\
\text { (second) }\end{array}$} \\
\hline & & & & $\begin{array}{c}\text { Full } \\
\text { enumeration } \\
\text { algorithm }\end{array}$ & VNS-PA & $\begin{array}{c}\text { Full } \\
\text { enumeration } \\
\text { algorithm } \\
\end{array}$ & VNS-PA \\
\hline TSS1 & $5 \times 1 \times 1$ & 1 & $\begin{array}{l}-0.152 \\
-0.514\end{array}$ & $\begin{array}{l}(69.6370,34.5699) \\
(54.8004,5.1013)\end{array}$ & $\begin{array}{l}(69.6370,34.5699) \\
(54.8004,5.1013)\end{array}$ & 0.0936 & 0.2340 \\
\hline TSS2 & $7 \times 1 \times 1$ & 1 & $\begin{array}{l}-0.152 \\
-0.514\end{array}$ & $\begin{array}{l}(94.9153,113.8748) \\
(63.0024,31.9535)\end{array}$ & $\begin{array}{l}(94.9153,113.8748) \\
(63.0024,31.9535)\end{array}$ & 1.3572 & 0.3120 \\
\hline TSS3 & $10 \times 1 \times 1$ & 1 & $\begin{array}{l}-0.152 \\
-0.514\end{array}$ & $\begin{array}{l}(151.2996,351.0591) \\
(90.7567,108.1328)\end{array}$ & $\begin{array}{l}(151.2996,351.0591) \\
(90.7567,108.1328)\end{array}$ & 1051.8679 & 0.3900 \\
\hline TSS4 & $5 \times 1 \times 2$ & 1 & $\begin{array}{l}-0.152 \\
-0.514\end{array}$ & $\begin{array}{l}(169.4504,157.2585) \\
(124.8355,26.0678)\end{array}$ & $\begin{array}{l}(169.4504,157.2585) \\
(124.8355,26.0678)\end{array}$ & 0.0936 & 0.2340 \\
\hline TSS5 & $7 \times 1 \times 2$ & 1 & $\begin{array}{l}-0.152 \\
-0.514\end{array}$ & $\begin{array}{l}(211.8133,444.9392) \\
(140.0740,151.4606)\end{array}$ & $\begin{array}{l}(211.8133,444.9392) \\
(140.0740,151.4606)\end{array}$ & 1.7784 & 0.4680 \\
\hline TSS6 & $10 \times 1 \times 2$ & 1 & $\begin{array}{l}-0.152 \\
-0.514\end{array}$ & $\begin{array}{l}(312.2389,1396.4222) \\
(190.6457,531.0124)\end{array}$ & $\begin{array}{l}(311.4924,1399.5332) \\
(190.6457,531.0124)\end{array}$ & 1450.0448 & 0.4992 \\
\hline TSS7 & $5 \times 1 \times 1$ & 3 & $\begin{array}{l}-0.152 \\
-0.514\end{array}$ & $\begin{array}{l}(30.6000,57.4089) \\
(27.5042,51.2183)\end{array}$ & $\begin{array}{l}(30.6000,57.4089) \\
(27.5042,51.2183)\end{array}$ & 0.0780 & 0.6084 \\
\hline TSS8 & $7 \times 1 \times 1$ & 3 & $\begin{array}{l}-0.152 \\
-0.514\end{array}$ & $\begin{array}{l}(40.0856,58.8299) \\
(34.4744,39.8322)\end{array}$ & $\begin{array}{l}(40.0856,58.8299) \\
(34.4744,39.8322)\end{array}$ & 1.4664 & 2.0124 \\
\hline TSS9 & $10 \times 1 \times 1$ & 3 & $\begin{array}{l}-0.152 \\
-0.514\end{array}$ & $\begin{array}{l}(57.0319,205.4145) \\
(49.7259,135.9363)\end{array}$ & $\begin{array}{l}(57.0319,205.4145) \\
(49.7259,135.9363)\end{array}$ & 1154.5790 & 0.9360 \\
\hline TSS10 & $5 \times 1 \times 2$ & 3 & $\begin{array}{l}-0.152 \\
-0.514\end{array}$ & $\begin{array}{l}(69.1001,130.9594) \\
(61.3108,110.7873)\end{array}$ & $\begin{array}{l}(69.1001,130.9594) \\
(61.3108,110.7873)\end{array}$ & 0.1092 & 0.8736 \\
\hline TSS11 & $7 \times 1 \times 2$ & 3 & $\begin{array}{l}-0.152 \\
-0.514\end{array}$ & $\begin{array}{l}(82.8091,222.2887) \\
(68.6763,169.9968)\end{array}$ & $\begin{array}{l}(82.8091,222.2887) \\
(68.6763,169.9968)\end{array}$ & 2.0748 & 2.9484 \\
\hline TSS12 & $10 \times 1 \times 2$ & 3 & $\begin{array}{l}-0.152 \\
-0.514\end{array}$ & $\begin{array}{l}(119.0647,653.7478) \\
(96.9553,479.2494)\end{array}$ & $\begin{array}{l}(120.3475,651.8989) \\
(96.9553,479.2494)\end{array}$ & 1654.4686 & 1.7004 \\
\hline TSS13 & $5 \times 2 \times 1$ & 1,1 & $\begin{array}{l}-0.152 \\
-0.514\end{array}$ & $\begin{array}{l}(104.4372,87.9622) \\
(76.4417,26.4530)\end{array}$ & $\begin{array}{l}(104.4372,87.9622) \\
(76.4417,26.4530)\end{array}$ & 0.0936 & 0.4056 \\
\hline TSS14 & $7 \times 2 \times 1$ & 1,1 & $\begin{array}{l}-0.152 \\
-0.514\end{array}$ & $\begin{array}{l}(123.8755,172.4553) \\
(83.6435,42.2256)\end{array}$ & $\begin{array}{l}(123.8755,172.4553) \\
(83.6435,42.2256)\end{array}$ & 1.8720 & 0.4524 \\
\hline
\end{tabular}


Table 3. Details of special small-sized problems and results of algorithms (continued).

\begin{tabular}{|c|c|c|c|c|c|c|c|}
\hline \multirow{2}{*}{$\begin{array}{c}\text { Test } \\
\text { problem }\end{array}$} & \multirow{2}{*}{$n \times g \times L$} & \multirow{2}{*}{$m^{t}$} & \multirow{2}{*}{$a^{t}$} & \multicolumn{2}{|c|}{$\begin{array}{l}\text { Best combination } \\
\quad\left(f_{1}(x), f_{2}(x)\right)\end{array}$} & \multicolumn{2}{|c|}{$\begin{array}{c}\text { CPU time } \\
\text { (second) }\end{array}$} \\
\hline & & & & $\begin{array}{l}\text { Full } \\
\text { enumeration } \\
\text { algorithm }\end{array}$ & VNS-PA & $\begin{array}{c}\text { Full } \\
\text { enumeration } \\
\text { algorithm }\end{array}$ & VNS-PA \\
\hline TSS15 & $10 \times 2 \times 1$ & 1,1 & $\begin{array}{l}-0.152 \\
-0.514\end{array}$ & $\begin{array}{l}(171.3400,474.0023) \\
(108.4170,195.3698)\end{array}$ & $\begin{array}{l}(171.3400,474.0023) \\
(108.4170,195.3698)\end{array}$ & 1458.6249 & 0.6240 \\
\hline TSS16 & $5 \times 2 \times 2$ & 1,1 & $\begin{array}{l}-0.152 \\
-0.514\end{array}$ & $\begin{array}{l}(188.2462,311.5455) \\
(143.8270,143.7925)\end{array}$ & $\begin{array}{l}(188.2462,311.5455) \\
(143.8270,143.7925)\end{array}$ & 0.1248 & 0.4056 \\
\hline TSS17 & $7 \times 2 \times 2$ & 1,1 & $\begin{array}{l}-0.152 \\
-0.514\end{array}$ & $\begin{array}{l}(236.0987,824.1130) \\
(162.6190,442.2210)\end{array}$ & $\begin{array}{l}(236.0987,824.1130) \\
(162.6190,442.2210)\end{array}$ & 2.6988 & 0.6240 \\
\hline TSS18 & $10 \times 2 \times 2$ & 1,1 & $\begin{array}{l}-0.152 \\
-0.514\end{array}$ & $\begin{array}{l}(318.1379,1363.3069) \\
(199.6826,496.1035)\end{array}$ & $\begin{array}{l}(317.5031,1370.3175) \\
(199.6826,496.1035)\end{array}$ & 2238.1931 & 0.9360 \\
\hline TSS19 & $5 \times 2 \times 1$ & 3,3 & $\begin{array}{l}-0.152 \\
-0.514\end{array}$ & $\begin{array}{l}(54.3001,0) \\
(50.6050,0)\end{array}$ & $\begin{array}{l}(54.3001,0) \\
(50.6050,0)\end{array}$ & 0.1092 & 1.1544 \\
\hline TSS20 & $7 \times 2 \times 1$ & 3,3 & $\begin{array}{l}-0.152 \\
-0.514\end{array}$ & $\begin{array}{l}(61.6001,15.6742) \\
(54.7058,3.5283)\end{array}$ & $\begin{array}{l}(61.6001,15.6742) \\
(54.7058,3.5283)\end{array}$ & 2.0592 & 1.2948 \\
\hline TSS21 & $10 \times 2 \times 1$ & 3,3 & $\begin{array}{l}-0.152 \\
-0.514\end{array}$ & $\begin{array}{l}(78.3015,0) \\
(64.7782,0)\end{array}$ & $\begin{array}{l}(78.3015,0) \\
(64.7782,0)\end{array}$ & 1687.0104 & 1.2168 \\
\hline TSS22 & $5 \times 2 \times 2$ & 3,3 & $\begin{array}{l}-0.152 \\
-0.514\end{array}$ & $\begin{array}{l}(92.4000,8.9120) \\
(89.2045,8.9120)\end{array}$ & $\begin{array}{l}(92.4000,8.9120) \\
(89.2045,8.9120)\end{array}$ & 0.1248 & 2.8860 \\
\hline TSS23 & $7 \times 2 \times 2$ & 3,3 & $\begin{array}{l}-0.152 \\
-0.514\end{array}$ & $\begin{array}{l}(110.4704,20.1540) \\
(92.3819,20.1540)\end{array}$ & $\begin{array}{l}(110.4704,20.1540) \\
(92.3819,20.1540)\end{array}$ & 3.0576 & 1.5132 \\
\hline TSS24 & $10 \times 2 \times 2$ & 3,3 & $\begin{array}{l}-0.152 \\
-0.514\end{array}$ & $\begin{array}{l}(139.3810,46.0765) \\
(114.0321,10.2775)\end{array}$ & $\begin{array}{l}(139.3810,46.0765) \\
(114.0321,10.2775)\end{array}$ & 2713.5281 & 1.5132 \\
\hline
\end{tabular}

column, and the last column describes the average CPU time (second unit). The last two columns in these tables are applied to compare the results.

Time cost is an important factor when comparing different algorithms. According to a report in the last column of Tables 4 to 6 , the proposed algorithm is able to solve the small, medium, and large problems in the length of the interval [2.132, 14.0816], [37.4037, 204.8969], and [399.9735, 2740.6281] seconds, respectively. The SA algorithm has spent the interval from 7.1396 to $105.2512,476.0864$ to 2107.0976 , and 5372.7861 to 33238.1788 seconds for small, medium, and large problems, respectively. This result indicates that the proposed algorithm in comparison with other algorithm has a significant speed in solving the problem. In addition, Figures 3 to 5 plot the computational times of the two algorithms for small, medium, and large problems, respectively. It can be seen that the computational times or the running times of VNS-PA are considerably less than SA.

In order to evaluate the final solutions' quality of each algorithm, column ' $Z$ value' in Tables 4 to 6 is used. In fact, this column is the value of the proposed objective function (Eq. (42)). It is known that a new objective function is designed in terms of requests, comments, and viewpoints of the decision-makers. As noted, the seven additional traits are added to the proposed objective function. Consequently, the value 
Table 4. Results of VNS-PA and SA for small-sized problems.

\begin{tabular}{|c|c|c|c|c|c|c|c|c|}
\hline \multirow{2}{*}{$\begin{array}{c}\text { Test } \\
\text { problem }\end{array}$} & \multirow[t]{2}{*}{$\lambda$} & \multirow[t]{2}{*}{$a^{t}$} & \multicolumn{2}{|c|}{$\begin{array}{l}\text { Best combination } \\
\quad\left(f_{1}(x), f_{2}(x)\right)\end{array}$} & \multicolumn{2}{|c|}{$Z$ value } & \multicolumn{2}{|c|}{$\begin{array}{l}\text { CPU time } \\
\text { (second) }\end{array}$} \\
\hline & & & VNS-PA & SA & VNS-PA & SA & VNS-PA & SA \\
\hline \multirow{6}{*}{ TS1 } & \multirow{2}{*}{0.25} & -0.152 & $199.2667,10.7680$ & $199.2667,10.7680$ & 6 & 6 & \multirow{6}{*}{2.8886} & \multirow{6}{*}{7.1396} \\
\hline & & -0.514 & $143.0179,6.5738$ & $142.6272,6.5738$ & 2 & 7 & & \\
\hline & 0.50 & -0.152 & $199.4227,10.7680$ & $199.2667,10.7680$ & 2 & 7 & & \\
\hline & & -0.514 & $142.7290,6.5738$ & $142.9777,6.5738$ & 7 & 2 & & \\
\hline & \multirow{2}{*}{0.75} & -0.152 & $199.2667,10.7680$ & $199.2667,10.7680$ & 6 & 6 & & \\
\hline & & -0.514 & $142.6915,6.5738$ & $142.7923,6.5738$ & 7 & 2 & & \\
\hline \multirow{6}{*}{ TS2 } & \multirow{2}{*}{0.25} & -0.152 & $243.2911,481.2525$ & $250.0320,595.0765$ & 7 & 1 & \multirow{6}{*}{5.2598} & \multirow{6}{*}{22.9009} \\
\hline & & -0.514 & $143.8616,81.1516$ & $147.1509,81.1516$ & 7 & 2 & & \\
\hline & 050 & -0.152 & $243.9654,487.2905$ & $247.9816,584.7125$ & 7 & 1 & & \\
\hline & & -0.514 & $144.9413,79.9595$ & $148.4950,93.6028$ & 7 & 1 & & \\
\hline & \multirow{2}{*}{0.75} & -0.152 & $242.8082,479.3601$ & $244.7873,593.3016$ & 7 & 1 & & \\
\hline & & -0.514 & $147.0222,85.2628$ & $149.7693,110.9439$ & 7 & 1 & & \\
\hline \multirow{6}{*}{ TS3 } & \multirow{2}{*}{0.25} & -0.152 & $332.0071,654.2614$ & $341.5906,884.1664$ & 7 & 1 & \multirow{6}{*}{7.9742} & \multirow{6}{*}{54.9825} \\
\hline & & -0.514 & $177.2424,31.8050$ & $186.6698,31.8050$ & 7 & 2 & & \\
\hline & 0.50 & -0.152 & $329.9454,720.3330$ & $341.0588,963.2479$ & 7 & 1 & & \\
\hline & & -0.514 & $179.1097,31.8050$ & $184.8838,31.8050$ & 7 & 2 & & \\
\hline & \multirow{2}{*}{0.75} & -0.152 & $332.6917,709.4153$ & $333.4096,979.7004$ & 7 & 1 & & \\
\hline & & -0.514 & $179.2923,31.8050$ & $187.3393,35.1831$ & 7 & 1 & & \\
\hline \multirow{6}{*}{ TS4 } & \multirow{2}{*}{0.25} & -0.152 & $362.6511,669.0805$ & $366.2466,700.6363$ & 7 & 1 & \multirow{6}{*}{4.2354} & \multirow{6}{*}{13.2289} \\
\hline & & -0.514 & $235.3487,244.6433$ & $237.1159,251.1650$ & 7 & 1 & & \\
\hline & 0.50 & -0.152 & $362.6511,669.0805$ & $363.6052,680.2699$ & 7 & 1 & & \\
\hline & & -0.514 & $234.0973,241.5954$ & $237.1569,261.8670$ & 7 & 1 & & \\
\hline & \multirow{2}{*}{0.75} & -0.152 & $362.6511,669.0805$ & $363.7631,689.3951$ & 7 & 1 & & \\
\hline & & -0.514 & $234.0973,241.5954$ & $238.3404,256.7414$ & 7 & 1 & & \\
\hline & 0.25 & -0.152 & $503.5382,1917.2486$ & $510.9941,2092.8972$ & 7 & 1 & & \\
\hline & & -0.514 & $295.1834,375.1026$ & $298.6744,446.1804$ & 7 & 1 & & \\
\hline TS.5 & 0.50 & -0.152 & $500.6247,1959.2683$ & $510.6997,2120.0523$ & 7 & 1 & 7.3034 & 44.9906 \\
\hline & & -0.514 & $294.9953,379.0427$ & $296.5968,466.1450$ & 7 & 1 & & \\
\hline & 0.75 & -0.152 & $501.6289,1935.5674$ & $509.2380,2082.9364$ & 7 & 1 & & \\
\hline & & -0.514 & $296.9217,373.6401$ & $300.8927,475.7459$ & 7 & 1 & & \\
\hline & 0.25 & -0.152 & $580.4476,3694.0145$ & $594.6841,3940.5997$ & 7 & 1 & & \\
\hline & & -0.514 & $309.8356,592.1808$ & $311.6760,733.5276$ & 7 & 1 & & \\
\hline TS6 & 0.50 & -0.152 & $580.7073,3659.8599$ & $598.9998,3921.3354$ & 7 & 1 & 14.0816 & 105.2512 \\
\hline & & -0.514 & $310.8194,657.7603$ & $319.2303,771.8630$ & 7 & 1 & & \\
\hline & 0.75 & -0.152 & $582.6820,3671.9397$ & $590.5845,3872.5499$ & 7 & 1 & & \\
\hline & & -0.514 & $311.6901,633.1478$ & $324.2570,783.3545$ & 7 & 1 & & \\
\hline
\end{tabular}


Table 4. Results of VNS-PA and SA for small-sized problems (continued).

\begin{tabular}{|c|c|c|c|c|c|c|c|c|}
\hline \multirow{2}{*}{$\begin{array}{c}\text { Test } \\
\text { problem }\end{array}$} & \multirow[t]{2}{*}{$\lambda$} & \multirow[t]{2}{*}{$a^{t}$} & \multicolumn{2}{|c|}{$\begin{array}{l}\text { Best combination } \\
\quad\left(f_{1}(x), f_{2}(x)\right)\end{array}$} & \multicolumn{2}{|c|}{$Z$ value } & \multicolumn{2}{|c|}{$\begin{array}{l}\text { CPU time } \\
\text { (second) }\end{array}$} \\
\hline & & & VNS-PA & SA & VNS-PA & SA & VNS-PA & SA \\
\hline \multirow{6}{*}{ TS7 } & \multirow{2}{*}{0.25} & -0.152 & $260.8505,558.8386$ & $266.7315,586.6777$ & 7 & 1 & \multirow{6}{*}{2.1320} & \multirow{6}{*}{9.3835} \\
\hline & & -0.514 & $195.1480,271.7062$ & $197.4958,283.3652$ & 7 & 1 & & \\
\hline & 050 & -0.152 & $260.2963,565.4782$ & $263.4751,566.8055$ & 7 & 1 & & \\
\hline & 0.50 & -0.514 & $195.1480,271.7062$ & $198.6328,280.9816$ & 7 & 1 & & \\
\hline & \multirow{2}{*}{0.75} & -0.152 & $262.0414,576.1022$ & $264.9741,579.2656$ & 7 & 1 & & \\
\hline & & -0.514 & $195.1480,271.7062$ & $197.7790,278.6770$ & 7 & 1 & & \\
\hline \multirow{6}{*}{ TS8 } & \multirow{2}{*}{0.25} & -0.152 & $313.6088,1227.4819$ & $322.4067,1291.0516$ & 7 & 1 & \multirow{6}{*}{3.9572} & \multirow{6}{*}{31.6916} \\
\hline & & -0.514 & $215.9601,610.3063$ & $219.8523,663.4933$ & 7 & 1 & & \\
\hline & 0.50 & -0.152 & $312.5072,1222.1546$ & $319.5033,1314.4263$ & 7 & 1 & & \\
\hline & & -0.514 & $217.8121,607.9701$ & $221.4377,673.6174$ & 7 & 1 & & \\
\hline & \multirow{2}{*}{0.75} & -0.152 & $314.6037,1230.6496$ & $313.3079,1254.5750$ & 7 & 2 & & \\
\hline & & -0.514 & 216.9739609 .4153 & $219.6262,654.8639$ & 7 & 1 & & \\
\hline \multirow{6}{*}{ TS9 } & \multirow{2}{*}{0.25} & -0.152 & $362.7951,1944.6142$ & $383.3242,2185.8094$ & 7 & 1 & \multirow{6}{*}{6.9108} & \multirow{6}{*}{74.0407} \\
\hline & & -0.514 & $230.5568,701.5093$ & $234.1352,890.3517$ & 7 & 1 & & \\
\hline & 050 & -0.152 & $363.7038,1904.5234$ & $379.1131,2215.1135$ & 7 & 1 & & \\
\hline & 0.50 & -0.514 & $230.4182,700.7386$ & $243.1906,1044.9879$ & 7 & 1 & & \\
\hline & \multirow{2}{*}{0.75} & -0.152 & $362.5541,1887.7657$ & $385.0345,2275.0357$ & 7 & 1 & & \\
\hline & & -0.514 & $229.5163,706.7088$ & $233.2515,948.3872$ & 7 & 1 & & \\
\hline \multirow{6}{*}{ TS10 } & \multirow{2}{*}{0.25} & -0.152 & $424.3251,504.9937$ & $427.0749,522.4043$ & 7 & 1 & \multirow{6}{*}{3.4346} & \multirow{6}{*}{17.4851} \\
\hline & & -0.514 & $326.8493,212.4347$ & $328.2723,212.4347$ & 7 & 2 & & \\
\hline & 050 & -0.152 & $424.3251,504.9937$ & $418.7096,536.8717$ & 7 & 2 & & \\
\hline & 0.50 & -0.514 & $327.5276,212.6590$ & $327.5500,224.3122$ & 7 & 1 & & \\
\hline & \multirow{2}{*}{0.75} & -0.152 & $424.1367,513.3294$ & $425.3997,511.6717$ & 4 & 5 & & \\
\hline & & -0.514 & $326.8493,212.4347$ & $327.5500,224.3122$ & 7 & 1 & & \\
\hline
\end{tabular}

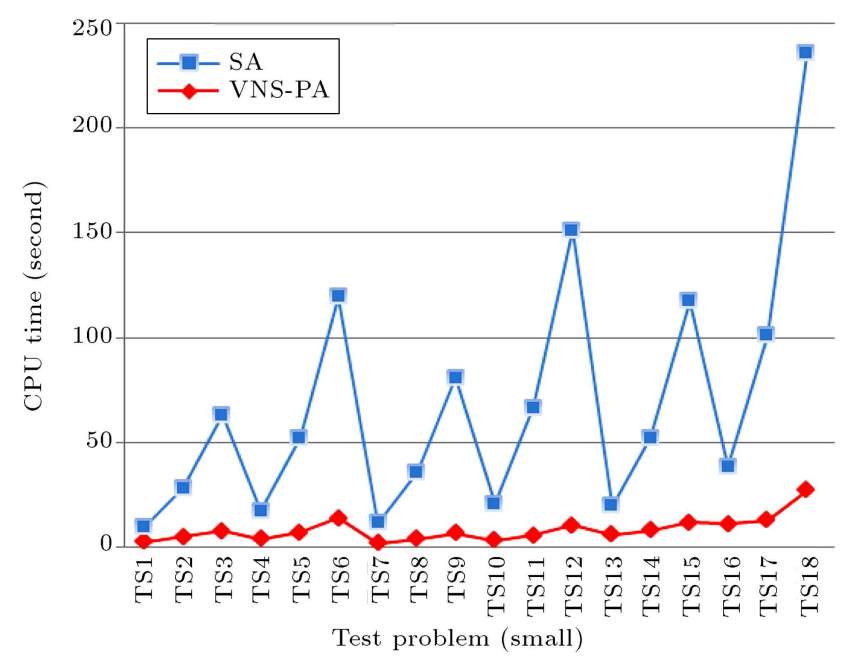

Figure 3. The computational times of VNS-PA and SA for small-sized problems.

of the proposed objective function demonstrates the number of provisions satisfied. It is obvious from this column that the proposed algorithm produces solutions more acceptable than others to the decision-maker in most cases.

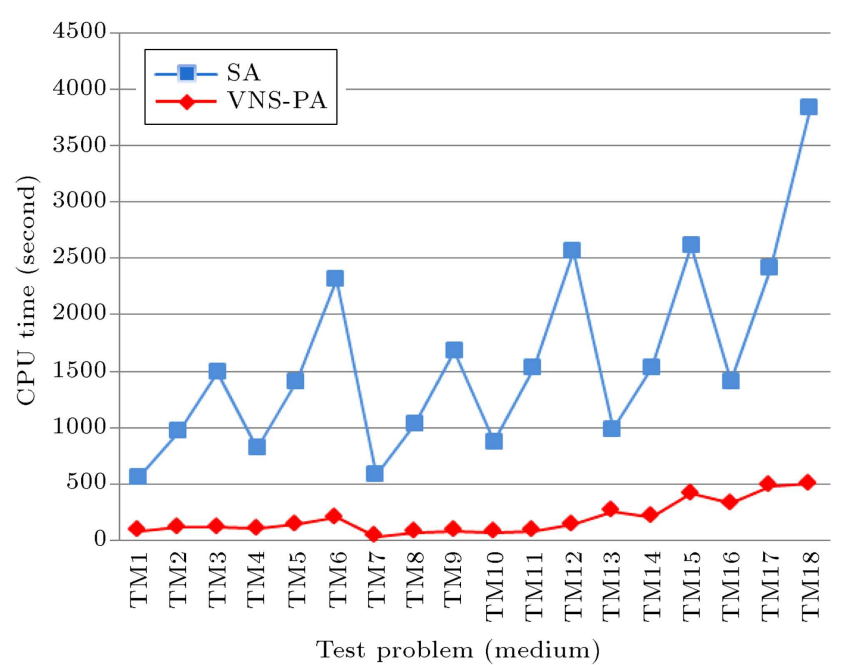

Figure 4. The computational times of VNS-PA and SA for medium-sized problems.

A graphical representation is provided to demonstrate output results of the VNS-PA and SA (Figure 6). This figure shows the obtained solutions of VNSPA and SA algorithms over twenty runs for TM8 problem. It is observed in this figure that the obtained 
Table 5. Results of VNS-PA and SA for medium-sized problems.

\begin{tabular}{|c|c|c|c|c|c|c|c|c|}
\hline \multirow{2}{*}{$\begin{array}{c}\text { Test } \\
\text { problem }\end{array}$} & \multirow[t]{2}{*}{$\lambda$} & \multirow[t]{2}{*}{$a^{t}$} & \multicolumn{2}{|c|}{$\begin{array}{l}\text { Best combination } \\
\qquad\left(f_{1}(x), f_{2}(x)\right)\end{array}$} & \multicolumn{2}{|c|}{$Z$ value } & \multicolumn{2}{|c|}{$\begin{array}{l}\text { CPU time } \\
\text { (second) }\end{array}$} \\
\hline & & & VNS-PA & SA & VNS-PA & SA & VNS-PA & SA \\
\hline \multirow{6}{*}{ TM1 } & \multirow{2}{*}{0.25} & -0.152 & $1358.0072,3408.2470$ & $1392.3175,481.9847$ & 7 & 1 & \multirow{6}{*}{87.8207} & \multirow{6}{*}{476.0864} \\
\hline & & -0.514 & $710.7291,501.9413$ & $713.8106,631.1734$ & 7 & 1 & & \\
\hline & 0.50 & -0.152 & $1349.8365,3508.6732$ & $1362.9074,4649.9621$ & 7 & 1 & & \\
\hline & & -0.514 & $697.8702,426.6741$ & $707.7943,543.0072$ & 7 & 1 & & \\
\hline & \multirow{2}{*}{0.75} & -0.152 & $1346.9045,3455.4110$ & $1434.2429,4270.7684$ & 7 & 1 & & \\
\hline & & -0.514 & $699.9794,469.6221$ & $728.7012,569.8631$ & 7 & 1 & & \\
\hline \multirow{6}{*}{ TM2 } & \multirow{2}{*}{0.25} & -0.152 & $1587.5586,9912.9650$ & $1640.0403,11376.7544$ & 7 & 1 & \multirow{6}{*}{117.6221} & \multirow{6}{*}{850.3614} \\
\hline & & -0.514 & $774.8318,668.1974$ & $792.7250,836.9903$ & 7 & 1 & & \\
\hline & 0.50 & -0.152 & $1583.9364,9938.3027$ & $1603.5932,11021.8430$ & 7 & 1 & & \\
\hline & & -0.514 & $768.0317,662.0809$ & $783.4835,974.5351$ & 7 & 1 & & \\
\hline & \multirow{2}{*}{0.75} & -0.152 & $1581.4487,9819.7511$ & $1602.7701,11238.9363$ & 7 & 1 & & \\
\hline & & -0.514 & $763.4621,636.2393$ & $782.8800,963.8487$ & 7 & 1 & & \\
\hline \multirow{6}{*}{ TM3 } & \multirow{2}{*}{0.25} & -0.152 & $1722.3264,13691.9690$ & $1761.4714,15302.3643$ & 7 & 1 & \multirow{6}{*}{116.4781} & \multirow{6}{*}{1375.3333} \\
\hline & & -0.514 & $820.5522,718.4882$ & $823.5066,1110.0868$ & 7 & 1 & & \\
\hline & 0.50 & -0.152 & $1706.7040,14002.9992$ & $1762.8476,15906.2309$ & 7 & 1 & & \\
\hline & & -0.514 & $801.1297,722.5415$ & $809.7602,1183.7418$ & 7 & 1 & & \\
\hline & \multirow{2}{*}{0.75} & -0.152 & $1719.8725,13576.8224$ & $1737.8602,15816.1517$ & 7 & 1 & & \\
\hline & & -0.514 & $783.5111,694.4817$ & $827.36237,1129.0669$ & 7 & 1 & & \\
\hline \multirow{6}{*}{ TM4 } & \multirow{2}{*}{0.25} & -0.152 & $1937.5971,8154.9848$ & $1968.6501,9273.4001$ & 7 & 1 & \multirow{6}{*}{106.3900} & \multirow{6}{*}{716.3851} \\
\hline & & -0.514 & $1032.5873,273.7329$ & $1046.2364,312.6222$ & 6 & 0 & & \\
\hline & 0.50 & -0.152 & $1954.6837,7906.0740$ & $1982.2601,9396.6521$ & 7 & 1 & & \\
\hline & & -0.514 & $1027.0291,298.2926$ & $1042.9893,335.9817$ & 7 & 1 & & \\
\hline & \multirow{2}{*}{0.75} & -0.152 & $1955.8740,7955.7180$ & $1987.5603,9347.4207$ & 7 & 1 & & \\
\hline & & -0.514 & $1014.2753,300.3054$ & $1034.9589,340.0318$ & 7 & 1 & & \\
\hline & 0.25 & -0.152 & $2171.8973,6938.9349$ & $2204.3881,8577.7483$ & 7 & 1 & & \\
\hline & & -0.514 & $1043.3988,220.3711$ & $1056.2580,289.8670$ & 7 & 1 & & \\
\hline TM5 & 0.50 & -0.152 & $2165.8051,7271.1548$ & $2198.4409,8270.3331$ & 7 & 1 & 141.5215 & 1270.6281 \\
\hline & & -0.514 & $1024.2842,237.0994$ & $1061.0080,321.4008$ & 7 & 1 & & \\
\hline & 0.75 & -0.152 & $2173.9788,7475.9645$ & $2204.3881,8577.74837$ & 7 & 1 & & \\
\hline & & -0.514 & $1056.6051,270.4899$ & $1055.0798,316.3902$ & 7 & 2 & & \\
\hline & 0.25 & -0.152 & $2448.8390,23631.9113$ & $2445.2614,25464.7873$ & 7 & 2 & & \\
\hline & & -0.514 & $1107.9278,1388.5805$ & $1133.5322,1718.9054$ & 7 & 1 & & \\
\hline TM6 & 0.50 & -0.152 & $2407.8444,23519.0797$ & $2461.6720,25849.3456$ & 7 & 1 & 204.8969 & 2107.0976 \\
\hline & & -0.514 & $1082.7561,1285.7236$ & $1127.3429,747.7833$ & 7 & 1 & & \\
\hline & 0.75 & -0.152 & $2412.1321,23692.2439$ & $2457.4201,25693.1264$ & 7 & 1 & & \\
\hline & & -0.514 & $1089.9052,1343.1999$ & $1139.7435,1678.2789$ & 7 & 1 & & \\
\hline
\end{tabular}


Table 5. Results of VNS-PA and SA for medium-sized problems (continued).

\begin{tabular}{|c|c|c|c|c|c|c|c|c|}
\hline \multirow{2}{*}{$\begin{array}{c}\text { Test } \\
\text { problem }\end{array}$} & \multirow[t]{2}{*}{$\lambda$} & \multirow[t]{2}{*}{$a^{t}$} & \multicolumn{2}{|c|}{$\begin{array}{l}\text { Best combination } \\
\quad\left(f_{1}(x), f_{2}(x)\right)\end{array}$} & \multicolumn{2}{|c|}{$Z$ value } & \multicolumn{2}{|c|}{$\begin{array}{l}\text { CPU time } \\
\text { (second) }\end{array}$} \\
\hline & & & VNS-PA & SA & VNS-PA & SA & VNS-PA & SA \\
\hline \multirow{6}{*}{ TM7 } & \multirow{2}{*}{0.25} & -0.152 & $1510.5763,5235.1305$ & $1543.1791,6681.8272$ & 7 & 1 & \multirow{6}{*}{37.4037} & \multirow{6}{*}{544.2458} \\
\hline & & -0.514 & $926.7607,1092.6267$ & $947.4376,1296.5893$ & 7 & 1 & & \\
\hline & 0.50 & -0.152 & $1510.9277,5398.6588$ & $1534.4259,6399.7784$ & 7 & 1 & & \\
\hline & \multirow{3}{*}{0.75} & -0.514 & $935.4230,1133.4456$ & $937.1943,1446.3046$ & 7 & 1 & & \\
\hline & & -0.152 & $1503.1919,5293.9026$ & $1543.0130,6749.0115$ & 7 & 1 & & \\
\hline & & -0.514 & $935.0112,1112.1764$ & $978.6252,1331.9636$ & 7 & 1 & & \\
\hline \multirow{6}{*}{ TM8 } & \multirow{2}{*}{0.25} & -0.152 & $1650.2158,9894.0778$ & $1687.5328,11584.0113$ & 7 & 1 & \multirow{6}{*}{73.9600} & \multirow{6}{*}{960.4357} \\
\hline & & -0.514 & $940.1271,1413.0879$ & $953.0611,2186.4549$ & 7 & 1 & & \\
\hline & 0.50 & -0.152 & $1639.7358,10091.1065$ & $1705.8712,11672.9533$ & 7 & 1 & & \\
\hline & & -0.514 & $952.4433,1412.7473$ & $987.2651,2345.4782$ & 7 & 1 & & \\
\hline & \multirow{2}{*}{0.75} & -0.152 & $1643.3910,9874.9129$ & $1686.6839,11686.5147$ & 7 & 1 & & \\
\hline & & -0.514 & $953.7051,1540.7814$ & $947.1948,2000.8256$ & 7 & 2 & & \\
\hline \multirow{6}{*}{ TM9 } & \multirow{2}{*}{0.25} & -0.152 & $1764.9555,12256.6683$ & $1828.3645,14726.4105$ & 7 & 1 & \multirow{6}{*}{87.3475} & \multirow{6}{*}{1592.3125} \\
\hline & & -0.514 & $942.2798,228.4465$ & $963.0248,510.0353$ & 7 & 1 & & \\
\hline & 0.50 & -0.152 & $1762.2872,13013.2504$ & $1829.1604,14726.9819$ & 7 & 1 & & \\
\hline & & -0.514 & $953.4593,280.4752$ & $934.6274,426.4481$ & 7 & 2 & & \\
\hline & \multirow{2}{*}{0.75} & -0.152 & $1774.8750,12595.7547$ & $1802.9873,14916.5601$ & 7 & 1 & & \\
\hline & & -0.514 & $934.2507,270.4154$ & $943.95911,341.4312$ & 7 & 1 & & \\
\hline \multirow{6}{*}{ TM10 } & \multirow{2}{*}{0.25} & -0.152 & $2162.4255,8277.5059$ & $2212.8799,9453.2599$ & 7 & 1 & \multirow{6}{*}{70.6346} & \multirow{6}{*}{803.4519} \\
\hline & & -0.514 & $1271.5181,1217.9128$ & $1289.9953,1516.7192$ & 7 & 1 & & \\
\hline & 0.50 & -0.152 & $2142.3687,8348.9381$ & $2220.2212,9744.1022$ & 7 & 1 & & \\
\hline & & -0.514 & $1273.4044,1034.0347$ & $1287.3140,1485.9365$ & 7 & 1 & & \\
\hline & \multirow{2}{*}{0.75} & -0.152 & $2147.2849,8014.8205$ & $2170.8716,9417.6667$ & 7 & 1 & & \\
\hline & & -0.514 & $1273.6014,1062.6905$ & $1278.8012,1555.42840$ & 7 & 1 & & \\
\hline
\end{tabular}

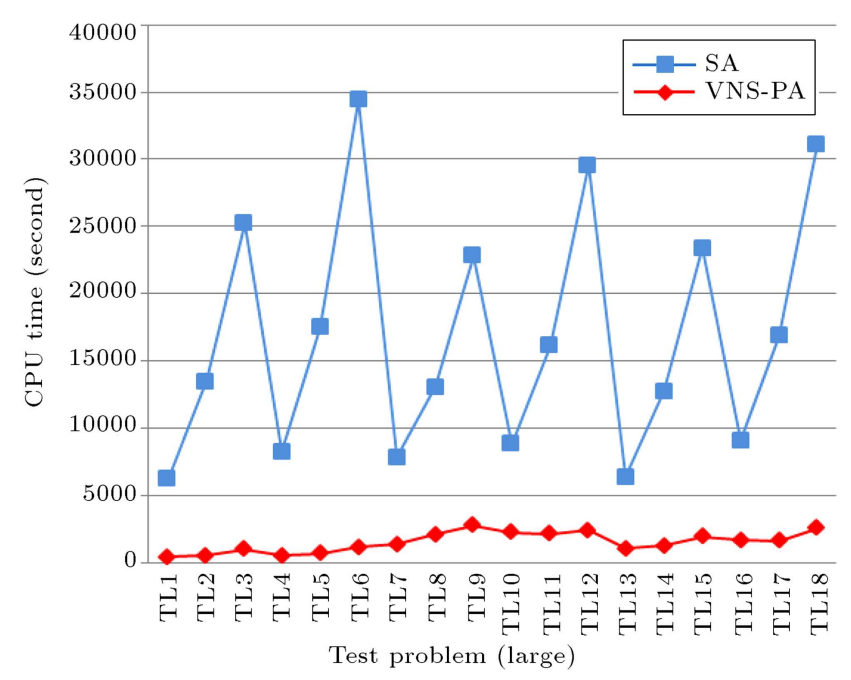

Figure 5. The computational times of VNS-PA and SA for large-sized problems.

solutions of SA algorithm have a trade-off between various objectives (first provision); however. other features (provisions 2 to 7 ) are not satisfied. This figure

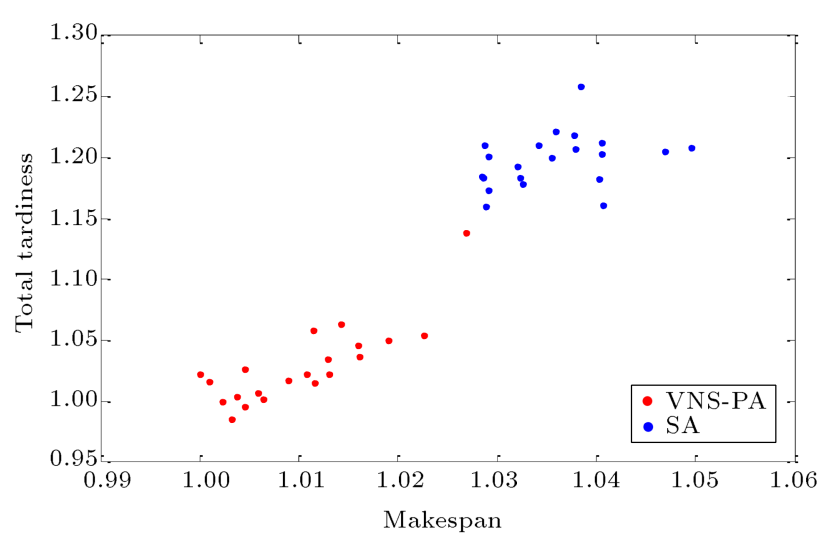

Figure 6. The generated combinations of VNS-PA and SA for TM8 problem.

illustrates and confirms the conclusion derived from the numerical results based on the performance criterion.

In order to visualize the performance of the two algorithms, the archived solutions from one run of each algorithm are selected to provide a graphical representation of the medium-sized problem (Figures 7 and 8). 
Table 6. Results of VNS-PA and SA for large-sized problems.

\begin{tabular}{|c|c|c|c|c|c|c|c|c|}
\hline \multirow{2}{*}{$\begin{array}{c}\text { Test } \\
\text { problem }\end{array}$} & \multirow[t]{2}{*}{$\lambda$} & \multirow[t]{2}{*}{$a^{t}$} & \multicolumn{2}{|c|}{$\begin{array}{l}\text { Best combination } \\
\qquad\left(f_{1}(x), f_{2}(x)\right)\end{array}$} & \multicolumn{2}{|c|}{$Z$ value } & \multicolumn{2}{|c|}{$\begin{array}{l}\text { CPU time } \\
\text { (second) }\end{array}$} \\
\hline & & & VNS-PA & SA & VNS-PA & $\mathbf{S A}$ & VNS-PA & SA \\
\hline \multirow{6}{*}{ TL1 } & \multirow{2}{*}{0.25} & -0.152 & $4164.8108,888.9346$ & $4150.5326,9707.1060$ & 7 & 2 & \multirow{6}{*}{399.9735} & \multirow{6}{*}{5815.6158} \\
\hline & & -0.514 & $2856.2347,1818.2137$ & $2980.6173,2696.4287$ & 7 & 1 & & \\
\hline & 0.50 & -0.152 & $4172.8403,7263.6107$ & $4140.6249,10448.5277$ & 7 & 2 & & \\
\hline & & -0.514 & $2851.1937,1701.7166$ & $2907.6958,2238.3060$ & 7 & 1 & & \\
\hline & \multirow{2}{*}{0.75} & -0.152 & $4122.6411,7589.2029$ & $4217.6346,9886.9807$ & 7 & 1 & & \\
\hline & & -0.514 & $2928.5115,1998.1715$ & $2969.8944,2597.4563$ & 7 & 1 & & \\
\hline \multirow{6}{*}{ TL2 } & \multirow{2}{*}{0.25} & -0.152 & $4924.4161,17103.1165$ & $5004.6500,21814.2270$ & 7 & 1 & \multirow{6}{*}{541.0842} & \multirow{6}{*}{12918.0241} \\
\hline & & -0.514 & $3047.7099,2757.4055$ & $3188.7915,4334.6762$ & 7 & 1 & & \\
\hline & 0.50 & -0.152 & $4844.7794,17346.8093$ & $4995.0974,22155.2641$ & 7 & 1 & & \\
\hline & 0.00 & -0.514 & $3158.1293,3193.2478$ & $3208.1847,4551.9991$ & 7 & 1 & & \\
\hline & \multirow{2}{*}{0.75} & -0.152 & $4921.2941,17406.0465$ & $4972.8046,21791.4472$ & 7 & 1 & & \\
\hline & & -0.514 & $3102.3326,3190.4566$ & $3207.5754,4061.7834$ & 7 & 1 & & \\
\hline \multirow{6}{*}{ TL3 } & \multirow{2}{*}{0.25} & -0.152 & $5385.8624,42011.7753$ & $5414.6467,50347.1421$ & 7 & 1 & \multirow{6}{*}{977.5698} & \multirow{6}{*}{24252.7370} \\
\hline & & -0.514 & $3034.6765,4181.8869$ & $3124.8720,6168.7497$ & 7 & 1 & & \\
\hline & 0.50 & -0.152 & $5319.1827,41937.1809$ & $5377.7320,51036.0832$ & 7 & 1 & & \\
\hline & & -0.514 & $3037.3411,4772.6762$ & $3125.0102,6967.6517$ & 7 & 1 & & \\
\hline & \multirow{2}{*}{0.75} & -0.152 & $5328.2457,43404.9209$ & $5319.8241,48411.2397$ & 7 & 2 & & \\
\hline & & -0.514 & $3059.7908,4528.1794$ & $3081.9344,5357.8333$ & 7 & 1 & & \\
\hline \multirow{6}{*}{ TL4 } & \multirow{2}{*}{0.25} & -0.152 & $5238.0803,5178.5804$ & $5316.8773,6127.8794$ & 7 & 1 & \multirow{6}{*}{494.8195} & \multirow{6}{*}{7765.0485} \\
\hline & & -0.514 & $3976.6742,1855.2834$ & $4018.2125,2511.7471$ & 7 & 1 & & \\
\hline & 0.50 & -0.152 & $5194.1544,5089.7478$ & $5261.0029,7422.4110$ & 7 & 1 & & \\
\hline & & -0.514 & $3919.0030,1499.9295$ & $4079.9497,2698.6888$ & 7 & 1 & & \\
\hline & \multirow{2}{*}{0.75} & -0.152 & $5173.9558,5233.0017$ & $5334.2117,6677.6926$ & 7 & 1 & & \\
\hline & & -0.514 & $3970.9994,1765.2185$ & $4082.4257,2377.5890$ & 7 & 1 & & \\
\hline & 025 & -0.152 & $6184.4666,27404.6983$ & $6141.7311,31493.5056$ & 7 & 2 & & \\
\hline & & -0.514 & $3870.8087,5512.8676$ & $4026.8506,6756.6762$ & 7 & 1 & & \\
\hline TL 5 & 0.50 & -0.152 & $6101.3901,28724.4902$ & $6143.6248,31619.7075$ & 7 & 1 & 6503225 & 16836.2180 \\
\hline & & -0.514 & $3877.0002,5345.2717$ & $4026.8506,6756.6762$ & 7 & 1 & & \\
\hline & 0.75 & -0.152 & $6117.5492,26892.2582$ & $6161.3233,32119.4781$ & 7 & 1 & & \\
\hline & & -0.514 & $3856.9911,5917.8244$ & $4030.7581,6761.5855$ & 7 & 1 & & \\
\hline & 0.25 & -0.152 & $6766.6240,43597.5278$ & $6888.2484,54867.6239$ & 7 & 1 & & \\
\hline & & -0.514 & $3900.2427,5025.0192$ & $4045.9839,7483.6438$ & 7 & 1 & & \\
\hline TL6 & 0.50 & -0.152 & $6709.1413,44029.4327$ & $6904.8833,54139.0280$ & 7 & 1 & 11439994 & 33238.1788 \\
\hline & & -0.514 & $3958.8094,5488.0653$ & $4045.9839,7483.6438$ & 7 & 1 & & \\
\hline & 0.75 & -0.152 & $6718.5627,44178.5029$ & $6822.2832,54204.3280$ & 7 & 1 & & \\
\hline & & -0.514 & $3921.3526,5248.3671$ & $4024.8998,7083.6322$ & 7 & 1 & & \\
\hline
\end{tabular}


Table 6. Results of VNS-PA and SA for large-sized problems (continued).

\begin{tabular}{|c|c|c|c|c|c|c|c|c|}
\hline \multirow{2}{*}{$\begin{array}{c}\text { Test } \\
\text { problem }\end{array}$} & \multirow[t]{2}{*}{$\lambda$} & \multirow[t]{2}{*}{$a^{t}$} & \multicolumn{2}{|c|}{$\begin{array}{l}\text { Best combination } \\
\quad\left(f_{1}(x), f_{2}(x)\right)\end{array}$} & \multicolumn{2}{|c|}{$Z$ value } & \multicolumn{2}{|c|}{$\begin{array}{l}\text { CPU time } \\
\text { (second) }\end{array}$} \\
\hline & & & VNS-PA & SA & VNS-PA & SA & VNS-PA & SA \\
\hline \multirow{6}{*}{ TL7 } & \multirow{2}{*}{0.25} & -0.152 & $6246.4658,45464.4212$ & $6446.4307,53745.4553$ & 7 & 1 & \multirow{6}{*}{1364.8942} & \multirow{6}{*}{6464.4941} \\
\hline & & -0.514 & $3406.0145,8823.4279$ & $3554.3961,11925.3353$ & 7 & 1 & & \\
\hline & 0.50 & -0.152 & $6237.0777,45944.7375$ & $6392.7104,54142.9377$ & 7 & 1 & & \\
\hline & U.Ju & -0.514 & $3396.0878,8714.8155$ & $3542.5495,11865.5127$ & 7 & 1 & & \\
\hline & \multirow{2}{*}{0.75} & -0.152 & $6249.8465,46375.5315$ & $6461.7802,54677.5356$ & 7 & 1 & & \\
\hline & & -0.514 & $3350.7780,8626.9597$ & $3593.1496,11313.1816$ & 7 & 1 & & \\
\hline \multirow{6}{*}{ TL8 } & \multirow{2}{*}{0.25} & -0.152 & $7799.8223,102904.7309$ & $8053.6935,111465.6123$ & 7 & 1 & \multirow{6}{*}{2078.8277} & \multirow{6}{*}{10958.0197} \\
\hline & & -0.514 & $3504.3149,10464.9499$ & $3584.2051,13289.3836$ & 7 & 1 & & \\
\hline & 0.50 & -0.152 & $7768.6782,103675.1357$ & $7985.6674,112430.6504$ & 7 & 1 & & \\
\hline & 0.50 & -0.514 & $3453.8425,10735.9776$ & $3598.5267,13416.4172$ & 7 & 1 & & \\
\hline & \multirow{2}{*}{0.75} & -0.152 & $7808.7714,105051.7819$ & $7896.8719,110282.3235$ & 7 & 1 & & \\
\hline & & -0.514 & $3438.1813,10881.6987$ & $3509.7262,12156.9936$ & 7 & 1 & & \\
\hline \multirow{6}{*}{ TL9 } & \multirow{2}{*}{0.25} & -0.152 & $8608.6794,140797.9846$ & $8737.4097,150944.8927$ & 7 & 1 & \multirow{6}{*}{2740.6281} & \multirow{6}{*}{20061.9885} \\
\hline & & -0.514 & $3614.3819,4511.8407$ & $3643.3332,5593.0288$ & 7 & 1 & & \\
\hline & 0.50 & -0.152 & $8717.7292,144991.9183$ & $8788.1980,150679.6168$ & 7 & 1 & & \\
\hline & & -0.514 & $3593.1215,3818.4760$ & $3643.3332,5593.0288$ & 7 & 1 & & \\
\hline & \multirow{2}{*}{0.75} & -0.152 & $8615.7452,143325.4396$ & $8826.6965,154839.6608$ & 7 & 1 & & \\
\hline & & -0.514 & $3582.6382,4139.2756$ & $3703.5108,5861.5393$ & 7 & 1 & & \\
\hline \multirow{6}{*}{ TL10 } & \multirow{2}{*}{0.25} & -0.152 & $8502.1664,60745.7984$ & $8709.4954,67947.5738$ & 7 & 1 & \multirow{6}{*}{2219.3429} & \multirow{6}{*}{6614.1875} \\
\hline & & -0.514 & $4444.4086,4366.3965$ & $4499.0049,5103.3440$ & 7 & 1 & & \\
\hline & 0.50 & -0.152 & $8529.3879,62572.9611$ & $8726.9028,67608.7707$ & 7 & 1 & & \\
\hline & 0.00 & -0.514 & $4424.8976,4036.2299$ & $4618.7833,6002.6988$ & 7 & 1 & & \\
\hline & \multirow{2}{*}{0.75} & -0.152 & $8501.4271,58917.8473$ & $8665.4233,68698.1919$ & 7 & 1 & & \\
\hline & & -0.514 & $4407.0757,4226.1895$ & $4585.5883,5683.9189$ & 7 & 1 & & \\
\hline
\end{tabular}

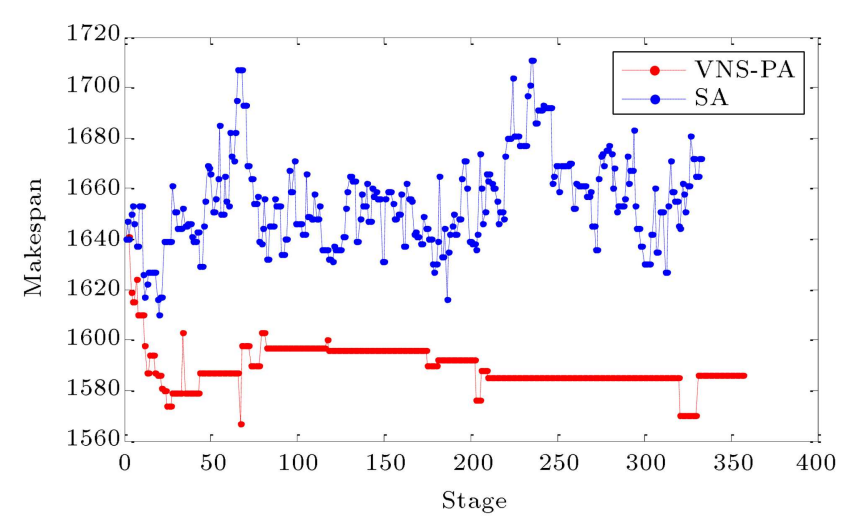

Figure 7. The makespan of archived solutions of TM2 problem.

Figures 7 and 8 represent makespan and the total tardiness graphs, respectively. It must be said that the VNS-PA and SA procedures begin with an identical initial solution. It is observed in these figures that the accepted solutions of VNS-PA algorithm are situated

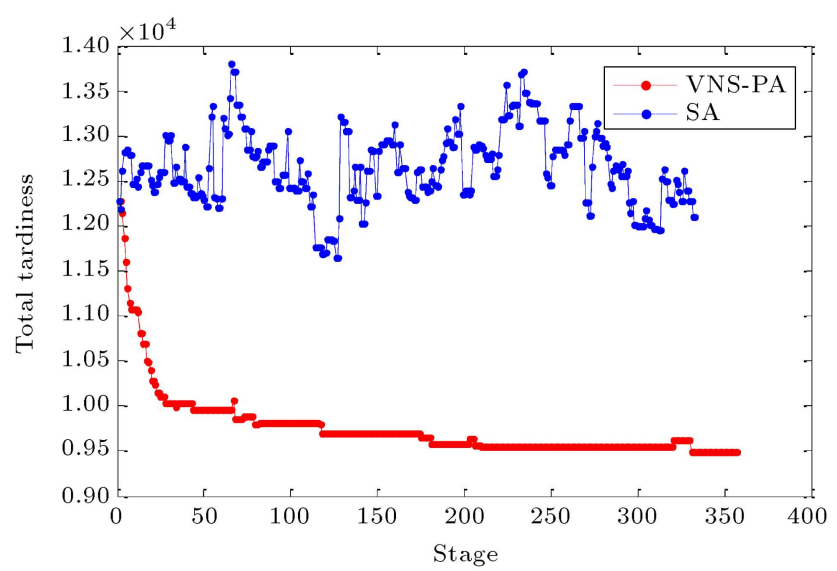

Figure 8. The total tardiness of the archived solutions of TM2 problem.

below the values obtained by the SA algorithm. As can be seen, the proposed algorithm is capable of providing better solutions than SA in terms of quality. Consequently, VNS-PA is more effective in minimizing 
the makespan and total tardiness for the RHFS with setup times and learning effect than the SA proposed by Mousavi et al. [12].

\section{Conclusion and further researches}

This paper considers the problem of scheduling jobs in a hybrid flow shop with the objectives of minimizing both the makespan and total tardiness, where the re-entrant line, setup times and position-dependent learning effects are considered. To describe the problem, first, a 0-1 MIP model was presented; then, the meta-heuristic method was applied to solve this problem, which belongs to NP-hard class. The solution procedure was categorized as an a priori approach. To the best of our knowledge, the approach used to solve the proposed problem has never been investigated in the scheduling problems. To demonstrate the validity of the proposed algorithm, the experiments were conducted on special small-sized problems. To show the efficiency and effectiveness of the proposed algorithm, computational experiments were carried out on various test problems. Computational results show that the proposed algorithm has very high reliability (excellent performance) and a significant speed to solve the problems. This research can be extended to several directions. First, it can be extended to the scheduling jobs with other system constraints, which have not been included in this paper. Second, the mentioned problem can be solved by some other meta-heuristic approaches. Finally, algorithm for other criteria, such as the flow time, mean waiting time, and the maximum lateness, can be developed carefully.

\section{References}

1. Allahverdi, A., Ng, C.T., Cheng, T.C.E., and Kovalyov, M.Y. "A survey of scheduling problems with setup times or costs", European J. Oper. Res., 187(3), pp. 985-1032 (2008).

2. Lin, D. and Lee, C.K.M. "A review of the research methodology for the re-entrant scheduling problem", Int. J. Prod. Res., 49(8), pp. 2221-2242 (2011).

3. Biskup, D.A. "Single machine scheduling with learning considerations", European J. Oper. Res., 115(1), pp. 173-178 (1999).

4. Biskup, D.A. "A state-of-the-art review on scheduling with learning effects", European J. Oper. Res., 188(2), pp. 315-329 (2008).

5. Gupta, J.N.D. "Two-stage, hybrid flow shop scheduling problem", J. Oper. Res. Soc., 39(4), pp. 359-364 (1988).

6. Jungwattanakit, J., Reodecha, M., Chaovalitwongse, P., and Werner, F. "Algorithms for flexible flow shop problems with unrelated parallel machines, setup times, and dual criteria", Int. J. Adv. Manuf. Technol., 37(3), pp. 354-370 (2008).
7. Behnamian, J., Fatemi Ghomi, S.M.T., and Zandieh, M. "A multi-phase covering Pareto-optimal front method to multi-objective scheduling in a realistic hybrid flowshop using a hybrid metaheuristic", Expert Syst. Appl., 36(8), pp. 11057-11069 (2009).

8. Naderi, B., Zandieh, M., Khaleghi Ghoshe Balagh, A., and Roshanaei, V. "An improved simulated annealing for hybrid flowshops with sequence-dependent setup and transportation times to minimize total completion time and total tardiness", Expert Syst. Appl., 36(6), pp. 9625-9633 (2009).

9. Rashidi, E., Jahandar, M., and Zandieh, M. "An improved hybrid multi-objective parallel genetic algorithm for hybrid flow shop scheduling with unrelated parallel machines", Int. J. Adv. Manuf. Technol., 49(9), pp. 1129-1139 (2010).

10. Dugardin, F., Yalaoui, F., and Amodeo., L. "New multi-objective method to solve reentrant hybrid flow shop scheduling problem", European J. Oper. Res., 203(1), pp. 22-31 (2010).

11. Cho, H-M., Bae, S-J., Kim, J., and Jeong, I-J. "Biobjective scheduling for reentrant hybrid flow shop using Pareto genetic algorithm", Comput. Ind. Eng., 61(3), pp. 529-541 (2011).

12. Mousavi, S.M., Zandieh, M., and Yazdani, M. "A simulated annealing/local search to minimize the makespan and total tardiness on a hybrid flowshop", Int. J. Adv. Manuf. Technol., 64(1), pp. 369-388 (2012).

13. Mousavi, S.M., Mousakhani, M., and Zandieh, M. "Biobjective hybrid flow shop scheduling: a new local search", Int. J. Adv. Manuf. Technol., 64(5), pp. 933950 (2012).

14. Hakimzadeh Abyaneh, S. and Zandieh, M. "Biobjective hybrid flow shop scheduling with sequencedependent setup times and limited buffers", Int. J. Adv. Manuf. Technol., 58(1), pp. 309-325 (2012).

15. Pargar, F. and Zandieh, M. "Bi-criteria SDST hybrid flow shop scheduling with learning effect of setup times: water flow-like algorithm approach", Int. J. Prod. Res., 50(10), pp. 2609-2623 (2012).

16. Sheikh, S. "Multi-objective flexible flow lines with due window, time lag, and job rejection", Int. J. Adv. Manuf. Technol., 64(9), pp. 1423-1433 (2013).

17. Tadayon, B. and Salmasi, N. "A two-criteria objective function flexible flowshop scheduling problem with machine eligibility constraint", Int. J. Adv. Manuf. Technol., 64(5), pp. 1001-1015 (2013).

18. Behnamian, J. and Zandieh, M. "Earliness and tardiness minimizing on a realistic hybrid flowshop scheduling with learning effect by advanced metaheuristic", Arab. J. Sci. Eng., 38(5), pp. 1229-1242 (2013).

19. Fadaei, M. and Zandieh, M. "Scheduling a bi-objective hybrid flow shop with sequence-dependent family setup times using metaheuristics", Arab. J. Sci. Eng., 38(8), pp. 2233-2244 (2013). 
20. Jolai, F., Asefi, H., Rabiee, M., and Ramezani, P. "Biobjective simulated annealing approaches for no-wait two-stage flexible flow shop scheduling problem", Sci. Iran., 20(3), pp. 861-872 (2013).

21. Luo, H., Du, B., Huang, G.Q., Chen, H., and Li, $\mathrm{X}$. "Hybrid flow shop scheduling considering machine electricity consumption cost", Int. J. Prod. Econ, 146(2), pp. 423-439 (2013).

22. Tran., T.H. and Ng, K.M. "A Hybrid water flow algorithm for multi-objective flexible flow shop scheduling problem", Eng. Optim., 45(4), pp. 483-502 (2013).

23. Su, S., Yu, H., Wu, Z., and Tian, W. "A distributed coevolutionary algorithm for multiobjective hybrid flowshop scheduling problems", Int. J. Adv. Manuf. Technol, , 70(1), pp. 477-494 (2014).

24. Attar, S.F., Mohammadi, M., Tavakkoli-Moghaddam, R., and Yaghoubi, S. "Solving a new multi-objective hybrid flexible flowshop problem with limited waiting times and machine-sequence-dependent set-up time constraints", Int. J. Comput. Integr. Manuf., 27(5), pp. 450-469 (2014).

25. Wang, S. and Liu, M. "Two-stage hybrid flow shop scheduling with preventive maintenance using multiobjective tabu search method", Int. J. Prod. Res., 52(5), pp. 1495-1508 (2014).

26. Ying, K.C., Lin, S.W., and Wan, S.Y. "Bi-objective reentrant hybrid flowshop scheduling: an iterated Pareto greedy algorithm", Int. J. Prod. Res., 52(19), pp. 5735-5747 (2014).

27. Mousavi, S.M. and Zandieh, M. "An efficient hybrid algorithm for a bi-objectives hybrid flow shop scheduling", Intell Autom Soft Co (2016). DOI: 10.1080/10798587.2016.1261956 (2016).

\section{Biographies}

Seyyed Mostafa Mousavi obtained his BSc degree in Industrial Engineering at University of Science and Industry, Behshar Branch, Iran (1999-2003), and MSc degree in Industrial Engineering at Islamic Azad
University, Qazvin Branch, Iran (2006-2008). He obtained his $\mathrm{PhD}$ degree in Industrial Engineering from Mazandaran University of Science and Technology, Iran (2011-2016). Currently, he is an Assistant Professor at Industrial Engineering Department, Islamic Azad University, Nowshahr Branch, Iran. His research interests are production planning and scheduling, and applied operations research.

Iraj Mahdavi is a Professor of Industrial Engineering at Mazandaran University of Science and Technology, Babol, Iran. He received his $\mathrm{PhD}$ degree from India in Production Engineering. $\mathrm{He}$ is also a member of the editorial board of four journals. He has published over 300 research papers. His research interests include cellular manufacturing, digital management of industrial enterprises, intelligent operation management, and industrial strategy setting.

Javad Rezaeian is currently an Associate Professor of Industrial Engineering at Mazandaran University of Science and Technology. His research interests are in the general area of meta-heuristic algorithms, cellular manufacturing systems, and production planning and scheduling.

Mostafa Zandieh accomplished his BSc degree in Industrial Engineering at Amirkabir University of Technology, Tehran, Iran (1994-1998), and MSc degree in Industrial Engineering at Sharif University of Technology, Tehran, Iran (1998-2000). He obtained his $\mathrm{PhD}$ degree in Industrial Engineering from Amirkabir University of Technology, Tehran, Iran (2000-2006). Currently, he is an Associate Professor at Industrial Management Department, Shahid Beheshti University, Tehran, Iran. His research interests are production planning and scheduling, financial engineering, quality engineering, applied operations research, simulation, and artificial intelligence techniques in the areas of manufacturing systems design. 